\title{
The effects of heat exchange and fluid production on the ignition of a porous solid
}

\author{
A.A. Shah ${ }^{\mathrm{a}, *}$, J. Brindley ${ }^{\mathrm{b}}$, A.C. McIntosh ${ }^{\mathrm{c}}$, J. Rademacher ${ }^{\mathrm{d}}$ \\ ${ }^{a}$ Mathematics Department, Simon Fraser University, BC, Canada V5A 1S6

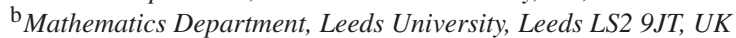 \\ ${ }^{\mathrm{c}}$ Energy and Resources Research Institute, Leeds University, Leeds LS2 9JT, UK \\ ${ }^{\mathrm{d}}$ Mathematics Department, University of British Columbia, BC, Canada V6T 1 Z2
}

Received 25 September 2005; accepted 14 December 2006

\begin{abstract}
In this paper we study a system of nonlinear parabolic equations representing the evolution of small perturbations in a model describing the combustion of a porous solid. The novelty of this system rests on allowing the fluid and solid phases to assume different temperatures, as opposed to the well-studied single-temperature model in which heat is assumed to be exchanged at an infinitely rapid rate. Moreover, the underlying model incorporates fluid creation, as a result of reaction, and this property is inherited by the perturbation system. With respect to important physico-chemical parameters we look for global and blowing-up solutions, both with and without heat loss and fluid production. In this context, blowup can be identified with thermal runaway, from which ignition of the porous solid is inferred (a self-sustaining combustion wave is generated). We then proceed to study the existence and uniqueness of a particular class of steady states and examine their relationship to the corresponding class of time-dependent problems. This enables us to extend the global-existence results, and to indicate consistency between the time-independent and time-dependent analyses. In order to better understand the effects of distinct temperatures in each phase, a number of our results are then compared with those of a corresponding single-temperature model. We find that the results coincide in the appropriate limit of infinite heat-exchange rate. However, when the heat exchange is finite the blowup results can be altered substantially.
\end{abstract}

(C) 2007 Elsevier Ltd. All rights reserved.

MSC: 80A25; 35K50; 35K57; 35J55

Keywords: Porous solid ignition; Dual temperature; Fluid production; Nonlinear parabolic; Global existence; Blowup; Steady states

\section{Introduction}

The ignition of combustible porous solids, inadvertently or otherwise (such as in self-propagating high-temperature synthesis, SHES), is an issue of great practical importance, particularly when such materials are capable of supporting a self-sustaining combustion wave that can cause their complete or partial degradation. As such, it has been the subject of a great number of theoretical studies, for example [2-7,9,12,13,16,17,19-21,23,34], and the many references therein. Few works have focused specifically on the ignition of porous solids, permeated by a fluid, though some exceptions are $[17,23,32,33,35,36]$. The majority of these make the assumption of temperature equilibrium between the solid and

\footnotetext{
* Corresponding author. Tel.: +1 778288 4292; fax: +1 6042686657

E-mail address: ashah@ pims.math.ca (A.A. Shah).
} 
fluid phases, resulting in a single equation for the temperature of the system. In general, this assumption is valid only if the rate of heat exchange between the phases is infinite (or at least asymptotically large in some sense), and it therefore neglects the role played by the heat exchange, different densities, heat transport properties and specific heat capacities of the two phases. It is the object of this paper to examine these processes in the simplest case of zeroth order reaction (no reactant consumption). Our approach considers small perturbations about an ambient state, resulting in a problem for the leading-order behaviour of the fluid and solid temperatures. We look for the "blowup" of the solutions (or "thermal runaway"), in which one of the temperatures becomes unbounded in a finite time. From this breakdown of the asymptotic model we can infer "ignition" of the porous solid, which from a physical point of view signifies the birth of a combustion wave. Mathematically, we must consider further terms in the asymptotic expansion to continue the solution in time. This approach to determining ignition bounds (and times) is well established and some of the seminal works in this area are due to Bebernes and Kassoy [5], Bellout [6] and Lacey [20], though much is owed to the work of Frank-Kemenetskii [12], Boddington et al. [7], Keller and Cohen [19], Aris [2], Fujita [14], and Kaplan [18].

A number of related questions that have received attention include the identification of the point (or set) at which blowup occurs, for example [13], and the continuation or not of the solution beyond the blowup time, usually in a weak sense, as in [21]. For combustion systems (with typically an exponential nonlinearity) the matchedasympotic approaches of Kapila [17], and Liñan and Williams [23] can elucidate the physical behaviour remarkably well. In particular, Kapila derives asymptotic solutions in each of the spatio-temporal regions that are present in the birth of a combustion wave through a porous energetic solid subjected to a constant heat flux. His approach rests on the assumption that ignition occurs before any non-negligible consumption of the (highly) energetic material has taken place. Unfortunately, the extension of this approach to the case of less energetic materials, where significant consumption occurs before the onset of ignition, has proved a formidable task in even the simplest model systems. Also of practical and theoretical interest is the propagation of the combustion wave that forms after ignition, for example [24-26,39]. Here we restrict ourselves to identifying conditions for blowup and global existence.

The outline of this paper is as follows. In the next section we derive the model. In Section 3 we treat the timedependent problem by first establishing a comparison lemma and local existence. We then look for blowup solutions in the special case in which no fluid is produced/consumed during reaction and no heat is lost from the system. The time-dependent problem with fluid production, both with and without heat loss, is examined in Section 5 . In Section 6 we study the existence and uniqueness of the class of steady states (zero heat loss and no fluid production). We are interested in the steady states as global attractors for the time-dependent problems. A discussion and summary follow in Section 7, where we also draw comparisons with the results for the single-temperature limit. We demonstrate that the dual- and single-temperature results coincide in the appropriate limit of infinite heat-exchange rate.

\section{Modelling and problem derivation}

Consider a bulk porous solid occupying a volume $\Omega$ (composed of both the solid and pore volumes). On the boundary, $\partial \Omega$, the material is maintained at an ambient constant temperature. We assume that an exothermic reaction takes place in the following form:

$$
\mathrm{Y} \longrightarrow \mathrm{F}+\mathrm{S}
$$

where $\mathrm{Y}$ represents a solid fuel and $\mathrm{F}$ and $\mathrm{S}$ are fluid and solid products, respectively. Examples of the gas and solid products are ash and carbon dioxide. In gas-less self-propagating high-temperature synthesis the fluid is liquid and components of the solid can undergo melting.

A system of equations representing combustion inside a porous medium can be found in $[32,33,38]$. Important assumptions underlying these models, and used here, are as follows:

(1) We assume that the oxygen supply is always adequate locally to support combustion; thus the reaction is not oxygen limited.

(2) We assume a single density, specific heat capacity and thermal conductivity for the fluid phase and similarly for the solid phase. 
(3) Separate energy balances are expressed for the fluid and solid phases, with a linear rate of interfacial heat transfer between the two. The density and thermal capacity of the solid are assumed to exceed those of the fluid, thus the energy released in the reaction is primarily spent on heating the solid. It is further assumed that the fluid is heated by the solid and by the impact of the fluid product at the temperature of the solid.

The equations can be written as follows:

$$
\begin{aligned}
& \rho_{\mathrm{s}}^{\prime} C_{\mathrm{s}}^{\prime}(1-\phi) \frac{\partial T_{\mathrm{s}}^{\prime}}{\partial t^{\prime}}-\lambda_{\mathrm{s}}^{\prime} \nabla^{\prime} \cdot\left((1-\phi) \nabla^{\prime} T_{\mathrm{s}}^{\prime}\right)=\underbrace{\rho_{\mathrm{s}}^{\prime} Q^{\prime} \mathscr{R}^{\prime}}_{\text {heat of reaction }}-\underbrace{\widetilde{\omega}^{\prime}\left(T_{\mathrm{s}}^{\prime}-T_{\mathrm{f}}^{\prime}\right)}_{\text {heat transfer }}-\underbrace{\widetilde{\alpha}^{\prime}\left(T_{\mathrm{s}}^{\prime}-T_{0}^{\prime}\right)}_{\text {bulk heat loss }}, \\
& \rho_{\mathrm{f}}^{\prime} C_{\mathrm{f}}^{\prime} \phi \frac{\partial T_{\mathrm{f}}^{\prime}}{\partial t^{\prime}}+\rho_{\mathrm{f}}^{\prime} \mathbf{u}_{\mathrm{f}}^{\prime} \nabla^{\prime} T_{\mathrm{f}}^{\prime}-\lambda_{\mathrm{f}}^{\prime} \nabla^{\prime} \cdot\left(\phi \nabla^{\prime} T_{\mathrm{f}}^{\prime}\right)=\underbrace{\left(T_{\mathrm{s}}^{\prime}-T_{\mathrm{f}}^{\prime}\right) \widetilde{v} \rho_{\mathrm{s}}^{\prime} C_{\mathrm{f}}^{\prime} \mathscr{R}^{\prime}}_{\text {heat content increase }}+\widetilde{\omega}^{\prime}\left(T_{\mathrm{s}}^{\prime}-T_{\mathrm{f}}^{\prime}\right), \\
& \frac{\partial \psi}{\partial t^{\prime}}=-\mathscr{R}^{\prime} \equiv \underbrace{-A \psi \mathrm{e}^{E_{\mathrm{a}}} / R^{\prime} T_{\mathrm{s}}^{\prime}}_{\text {solid reactant consumption }} \quad, \\
& \frac{\partial}{\partial t^{\prime}}\left(\rho_{\mathrm{f}}^{\prime} \phi\right)=\underbrace{\tilde{v} \rho_{\mathrm{s}}^{\prime} \mathscr{R}^{\prime}}_{\text {fluid production }}-\nabla^{\prime} \cdot\left(\rho_{\mathrm{f}}^{\prime} \mathbf{u}_{\mathrm{f}}^{\prime} \phi\right) \quad(\text { gas continuity), } \\
& \mathbf{u}_{\mathrm{f}}^{\prime}=-\frac{k^{\prime}}{\mu^{\prime}} \nabla^{\prime} P_{\mathrm{f}}^{\prime} \quad(\text { Darcy's Law }), \quad P_{\mathrm{f}}^{\prime}=P_{\mathrm{f}}^{\prime}\left(\rho_{\mathrm{f}}^{\prime}, T_{\mathrm{f}}^{\prime}\right) \quad \text { (equation of state) }, \\
& \widetilde{v}(1-\psi)=\phi-(1-\widetilde{v}) \phi_{0} \quad(\text { mass balance })
\end{aligned}
$$

with the boundary conditions:

$$
\left(T_{\mathrm{f}}^{\prime}, T_{\mathrm{s}}^{\prime}, \rho_{\mathrm{f}}^{\prime}\right)=\left(T_{0}^{\prime}, T_{0}^{\prime}, \rho_{\mathrm{g} 0}^{\prime}\right), \quad\left(x^{\prime}, t^{\prime}\right) \in \partial \Omega \times\left(0, \tau^{\prime}\right)
$$

and initial conditions:

$$
\left(T_{\mathrm{f}}^{\prime}, T_{\mathrm{s}}^{\prime}, \psi, \rho_{\mathrm{f}}^{\prime}\right)=\left(T_{\mathrm{f} 0}^{\prime}, T_{\mathrm{s} 0}^{\prime}, 1-\phi_{0}, \rho_{\mathrm{f} 0}^{\prime}\right), \quad x^{\prime} \in \Omega \subset \mathbb{R}^{n}, \quad t=0
$$

Here, $\psi$ is the volume fraction of $\mathrm{Y}, T_{\mathrm{f}}^{\prime}\left(T_{\mathrm{s}}^{\prime}\right)$ is the temperature of the fluid (solid) phase, $\phi$ is the porosity, $P_{\mathrm{f}}^{\prime}$ is fluid pressure, $\rho_{\mathrm{f}}^{\prime}\left(\rho_{\mathrm{s}}^{\prime}\right)$ is the (actual) density of the fluid (solid) phase and $\mathbf{u}_{\mathrm{f}}^{\prime}$ is the fluid velocity. The factor $\widetilde{v}$ represents the mass of fluid that is produced per unit mass of solid reactant. The first term on the right-hand side of Eq. (2.1a) represents heat generation by an irreversible one-step reaction based on the Arrhenius law, $\mathscr{R}^{\prime}$, with heat of reaction $Q^{\prime}$. The second term represents interfacial heat transfer and the third term is a volumetric heat loss, valid for particular geometries and representing, for example, heat loss in a lateral direction or to a coolant. The other quantities are defined in Table 1. Expression (2.1f) is arrived at by calculating, in each control volume, how much of the products of reaction are fluid and solid (see [33] for a derivation). The fluid product leaves the reaction zone at the temperature of the solid. We therefore place the term $-\rho_{\mathrm{s}}^{\prime} C_{\mathrm{s}}^{\prime} T_{\mathrm{s}}^{\prime} \partial_{t} \phi$ (arising from the solid energy equation) in the fluid energy equation $\left(C_{\mathrm{s}}^{\prime}\right.$ is replaced with $C_{\mathrm{g}}^{\prime}$ to ensure consistency). The resulting extra source term in the fluid energy equation represents the change in heat capacity of the fluid.

In both phases, the rates of heat exchange to the environment (or Biot numbers) are assumed to be infinite, which yields instantaneous relaxation to the same (ambient) temperature on $\partial \Omega$. 
Table 1

Dimensional parameters appearing in the initial-boundary value problem (2.1)

\begin{tabular}{lll}
\hline Quantity & Symbol & Units \\
\hline Pre-exponential factor & $A^{\prime}$ & $\mathrm{s}^{-1}$ \\
Heat of reaction & $Q^{\prime}$ & $\mathrm{J} \mathrm{kg}^{-1}$ \\
Activation energy & $E_{\mathrm{a}}^{\prime}$ & $\mathrm{J} \mathrm{kmol}^{-1}$ \\
Permeability & $k$ & $\mathrm{~kg} \mathrm{~m}^{2} \mathrm{~s}$ \\
Dynamic viscosity & $\mu^{\prime}$ & $\mathrm{kg} \mathrm{m}^{2} \mathrm{~s}$ \\
Heat-loss parameter & $\widetilde{\alpha}^{\prime}$ & $\mathrm{W} \mathrm{m}^{-3} \mathrm{~K}^{-1}$ \\
Specific heat capacity of solid & $C_{\mathrm{s}}^{\prime}$ & $\mathrm{J} \mathrm{kg}^{-1} \mathrm{~K}^{-1}$ \\
Specific heat capacity of fluid & $C_{\mathrm{f}}^{\prime}$ & $\mathrm{J} \mathrm{kg}^{-1} \mathrm{~K}^{-1}$ \\
Thermal conductivity of solid & $\lambda_{\mathrm{s}}^{\prime}$ & $\mathrm{W} \mathrm{m}^{-1} \mathrm{~K}^{-1}$ \\
Thermal conductivity of fluid & $\lambda_{\mathrm{f}}^{\prime}$ & $\mathrm{W} \mathrm{m}^{-1} \mathrm{~K}^{-1}$ \\
Mass of fluid produced per unit mass of reacted Y & $\widetilde{v}^{\prime}$ & - \\
Heat-exchange parameter & $\widetilde{\omega}^{\prime}$ & $\phi_{0}$ \\
Initial porosity & $T_{0}^{\prime}$ & - \\
Boundary temperature & $\rho_{\mathrm{f} 0}^{\prime}$ & $\mathrm{K}^{-1}$ \\
Fluid density at boundary & $T_{\mathrm{f}}^{\prime}\left(T_{\mathrm{s} 0}^{\prime}\right)$ & $\mathrm{K}$ \\
Initial fluid (solid) temperature & $R$ & $\mathrm{~J}$ \\
Universal gas constant & & $\mathrm{J} \mathrm{kmol}$ \\
\hline
\end{tabular}

To derive our perturbation problem it is convenient to non-dimensionalize Eqs. (2.1), according to the following:

$$
\begin{aligned}
& T_{\mathrm{s}}=\frac{T_{\mathrm{s}}^{\prime}}{T_{0}^{\prime}}, \quad T_{\mathrm{f}}=\frac{T_{\mathrm{f}}^{\prime}}{T_{0}^{\prime}}, \quad \rho_{\mathrm{f}}=\frac{\rho_{\mathrm{f}}^{\prime}}{\rho_{\mathrm{g} 0}^{\prime}}, \quad P_{\mathrm{f}}=\frac{P_{\mathrm{f}}^{\prime}}{P_{\mathrm{at}}^{\prime}}, \quad \mathbf{u}_{\mathrm{f}}=\frac{t_{\mathrm{c}}^{\prime} \mathbf{u}_{\mathrm{f}}^{\prime}}{x_{\mathrm{c}}^{\prime}}, \quad x=\frac{x^{\prime}}{x_{\mathrm{c}}^{\prime}}, \quad t=\frac{t^{\prime}}{t_{\mathrm{c}}^{\prime}}, \\
& E_{\mathrm{a}}=\frac{E_{\mathrm{a}}^{\prime}}{R^{\prime} T_{0}^{\prime}}, \quad t_{\mathrm{c}}^{\prime}=\frac{A^{\prime} \mathrm{e}^{E_{\mathrm{a}}}}{E_{\mathrm{a}}}, \quad x_{\mathrm{c}}^{\prime}=\sqrt{\frac{\lambda_{\mathrm{s}}^{\prime} t_{\mathrm{c}}^{\prime}}{\rho_{\mathrm{s}}^{\prime} C_{\mathrm{s}}^{\prime}}}, \quad Q=\frac{Q^{\prime}}{C_{\mathrm{s}}^{\prime} T_{\mathrm{a}}^{\prime}}, \quad \sigma=\frac{C_{\mathrm{f}}^{\prime}}{C_{\mathrm{s}}^{\prime}}, \\
& \delta=\frac{\rho_{\mathrm{g} 0}^{\prime}}{\rho_{\mathrm{s}}^{\prime}}, \quad k=\frac{\kappa^{\prime} t_{\mathrm{c}}^{\prime} P_{\mathrm{at}}^{\prime}}{\mu^{\prime} x_{\mathrm{c}}^{\prime}}, \quad \gamma=\frac{\lambda_{\mathrm{f}}^{\prime}}{\lambda_{\mathrm{s}}^{\prime}}, \quad \widetilde{\omega}=\frac{\widetilde{\omega}^{\prime} t_{\mathrm{c}}^{\prime}}{\rho_{\mathrm{s}}^{\prime} C_{\mathrm{s}}^{\prime}}, \quad \tilde{\alpha}=\frac{\widetilde{\alpha}^{\prime} t_{\mathrm{c}}^{\prime}}{\rho_{\mathrm{s}}^{\prime} C_{\mathrm{s}}^{\prime}}, \\
& T_{\mathrm{s} 0}=\frac{T_{\mathrm{s} 0}^{\prime}}{T_{0}^{\prime}}, \quad T_{\mathrm{f} 0}=\frac{T_{\mathrm{f} 0}^{\prime}}{T_{0}^{\prime}}, \quad \rho_{\mathrm{f} 0}=\frac{\rho_{\mathrm{f} 0}^{\prime}}{\rho_{\mathrm{f} 0}^{\prime}} .
\end{aligned}
$$

Here the characteristic time, $t_{\mathrm{c}}^{\prime}$, is a chemical reaction time and the characteristic length, $x_{\mathrm{c}}^{\prime}$, ensures a balance between the time scale of heat conduction in the solid and the chemical reaction time. With these definitions in place we obtain the following system:

$$
\begin{aligned}
& (1-\phi) \frac{\partial T_{\mathrm{s}}}{\partial t}-\nabla \cdot\left((1-\phi) \nabla T_{\mathrm{s}}\right)=Q \mathscr{R}-\widetilde{\omega}\left(T_{\mathrm{s}}-T_{\mathrm{f}}\right)-\widetilde{\alpha}\left(T_{\mathrm{s}}-1\right), \\
& \rho_{\mathrm{f}} \phi \frac{\partial T_{\mathrm{f}}}{\partial t}+\rho_{\mathrm{f}} \mathbf{u}_{\mathrm{f}} \nabla T_{\mathrm{f}}-\frac{\gamma}{\sigma \delta} \nabla \cdot\left(\phi \nabla T_{\mathrm{f}}\right)=\frac{\widetilde{v}}{\delta}\left(T_{\mathrm{s}}-T_{\mathrm{f}}\right) \mathscr{R}+\frac{\widetilde{\omega}}{\sigma \delta}\left(T_{\mathrm{s}}-T_{\mathrm{f}}\right), \\
& \frac{\partial \psi}{\partial t}=-\mathscr{R} \equiv-\frac{\psi \mathrm{e}^{E_{\mathrm{a}}-E_{\mathrm{a}} / T_{\mathrm{s}}}}{E_{\mathrm{a}}} \\
& \frac{\partial}{\partial t}\left(\rho_{\mathrm{f}} \phi\right)+\nabla \cdot\left(\rho_{\mathrm{f}} \mathbf{u}_{\mathrm{f}} \phi\right)=\frac{\widetilde{v}}{\delta} \mathscr{R}, \\
& \mathbf{u}_{\mathrm{f}}=-k \nabla P_{\mathrm{f}}, \quad P_{\mathrm{f}}=P_{\mathrm{f}}\left(\rho_{\mathrm{f}}, T_{\mathrm{f}}\right)
\end{aligned}
$$

with the boundary conditions:

$$
\left(T_{\mathrm{f}}, T_{\mathrm{s}}, \rho_{\mathrm{f}}\right)=(1,1,1), \quad(x, t) \in \partial \Omega \times(0, \tau)
$$


and initial conditions:

$$
\left(T_{\mathrm{f}}, T_{\mathrm{s}}, \psi, \rho_{\mathrm{f}}\right)=\left(T_{\mathrm{f} 0}, T_{\mathrm{s} 0}, 1-\phi_{0}, 1\right), \quad x \in \Omega, \quad t=0 .
$$

Eq. (2.1f) is unchanged. Note that the representation of the system using a continuum of two distinct temperatures, $T_{\mathrm{s}}^{\prime}$ and $T_{\mathrm{f}}^{\prime}$, assumes that the solid structure is fine-scale and interacting thermally with the fluid at all points. Of course, each temperature represents a local average in its respective phase. In the limit $\omega \rightarrow \infty, T_{\mathrm{S}} \rightarrow T_{\mathrm{f}}$ and Eqs. (2.1a) and (2.1b) may be combined to give a single equation, which we shall refer to as the "single-temperature equation":

$$
\frac{\partial}{\partial t} \underbrace{(\sigma \delta \phi T+(1-\phi) T)}_{\text {combined heat content }}-\nabla \cdot \underbrace{((1-\phi+\gamma \phi) \nabla T)}_{\text {combined heat flux }}=\frac{Q \psi \mathrm{e}^{E_{\mathrm{a}}-E_{\mathrm{a}} / T}}{E_{\mathrm{a}}}-\widetilde{\alpha}(T-1),
$$

where $T_{\mathrm{s}}=T_{\mathrm{f}}=T$. For small perturbations about an ambient state, under the Frank-Kemenetskii approximation, which we define below, this equation reduces to the classical Gelfand problem [4].

Of those appearing above, the dimensionless parameters that will be of most concern in this paper are $Q, \widetilde{\omega}, \gamma, \delta, \sigma, \widetilde{\alpha}$ and $\widetilde{v}$. We shall only consider $\widetilde{v} \geqslant 0$, so that there is either (i) net mass equilibrium $(\widetilde{v}=0$ ), or (ii) net mass creation $(\tilde{v}>0)$.

We make the assumption that in the early stages of ignition (before the birth of a combustion wave) the effects of heat and mass convection, variations in pressure and thermal expansion are weak in comparison to those of diffusion and chemical reaction. Experimental results suggest that this is an entirely reasonable approximation for many systems [8]. In SHES processes that require oxygen this assumption is not valid since the oxygen would need to be forced through the solid. Convective heat and mass transfer would then be non-negligible. To introduce the perturbation problem, we assume that the system is close to its ambient initial state (given by the initial-boundary conditions above), and introduce perturbations in the form:

$$
T_{\mathrm{S}} \sim 1+\frac{u}{E_{\mathrm{a}}}, \quad T_{\mathrm{f}} \sim 1+\frac{v}{E_{\mathrm{a}}}, \quad \psi \sim 1-\phi_{0}+\frac{w}{E_{\mathrm{a}}}, \quad T_{\mathrm{s} 0}=1+\frac{u_{o}}{E_{\mathrm{a}}}, \quad T_{\mathrm{f} 0}=1+\frac{v_{o}}{E_{\mathrm{a}}},
$$

where the dimensionless activation energy, $E_{\mathrm{a}} \gg 1$, is the perturbation parameter. After substituting these expansions into (2.1) and collecting terms of $O\left(E_{\mathrm{a}}^{-1}\right)$, we find that reactant consumption is effectively decoupled from the temperature problem, which is

$$
\begin{aligned}
& \frac{\partial u}{\partial t}-\Delta u=Q \mathrm{e}^{u}-\omega(u-v)-\alpha u, \\
& \frac{\partial v}{\partial t}-\beta \Delta v=v \mathrm{e}^{u}(u-v)+\kappa \omega(u-v)
\end{aligned}
$$

with boundary conditions

$$
(u, v)=(0,0), \quad(x, t) \in \partial \Omega \times(0, \tau)
$$

and initial conditions

$$
(u, v)=\left(u_{o}(x), v_{o}(x)\right), \quad x \in \Omega, \quad t=0,
$$

where

$$
\omega=\frac{\widetilde{\omega}}{1-\phi_{0}}, \quad \beta=\frac{\gamma}{\delta \sigma}, \quad v=\frac{\widetilde{v}\left(1-\phi_{0}\right)}{E_{\mathrm{a}} \delta \phi_{0}}, \quad \alpha=\frac{\tilde{\alpha}}{1-\phi_{0}}, \quad \kappa=\frac{1-\phi_{0}}{\sigma \delta \phi_{0}} .
$$

Note that we have used the so-called Frank-Kemenetskii approximation [12], which amounts to setting $E_{\mathrm{a}}^{-1}=0$ in the exponential reaction-rate term. The relative sizes of the terms appearing in Eqs. (2.6) depend on the material properties of the solid and fluid, through (2.7), and the nature of the reaction. It can happen that all terms balance and for generality we will assume throughout that they do. We return to this point in Section 7. 


\subsection{Notation and assumptions}

We use the symbol $C(\Omega)$ for functions which are continuous in a region $\Omega \subset \mathbb{R}^{n}$, and $C^{m}(\Omega)$ for functions with continuous partial derivatives up to and including order $m . C_{\alpha}(\Omega)$ denotes the space of Hölder continuous functions (exponent $\alpha \in(0,1)$ ), $L(\Omega)$ denotes Lipschitz continuous functions and $C^{m+\alpha}(\Omega)$ is used for functions that have Hölder continuous $m$ th order partial derivatives. The superscript loc, e.g. $L^{\text {loc }}(\Omega)$, is used for functions that belong to the superscripted space in a local sense, i.e., on any $\Omega^{\prime} \subset \subset \Omega$.

We use $W^{m, p}(\Omega)$ for functions that have $m$ th order distributional derivatives in the space $L^{p}(\Omega)$ of $p$ th Lesbesgueintegrable equivalence classes of functions.

For the parabolic domain $(0, \tau] \times \Omega \subset(0, \infty) \times \mathbb{R}^{n}$ we use the symbol $D_{\tau}$, while $S_{\tau}=(0, \tau] \times \partial \Omega$. The parabolic boundary is denoted $\Gamma_{\tau}=(\{0\} \times \Omega) \cup S_{\tau}$. The symbol $C^{k, m}\left(D_{\tau}\right)$ is used to denote the space of functions that have continuous time and spatial partial derivatives (in $D_{\tau}$ ) up to orders $k$ and $m$, respectively. Continuity in the closure of a set (for example $\overline{D_{\tau}}$ and $\bar{\Omega}$ ) is denoted similarly.

For each of these definitions we replace $C$ with $\mathbf{C}, L$ with $\mathbf{L}$ and $W$ with $\mathbf{W}$ for the corresponding space of 2-tuples. For example, $\mathbf{C}^{m}(\Omega) \equiv C^{m}(\Omega) \times C^{m}(\Omega)$.

We use the symbol $\mathbb{R}_{+}$to represent the non-negative portion of the real line, $[0, \infty)$. The symbol $\preccurlyeq$ is used for the total ordering of pairs of real numbers: $(a, b) \preccurlyeq(c, d)$ if $a \leqslant c$ and $b \leqslant d$, where $a, b, c, d \in \mathbb{R}$ and $\leqslant$ is the usual order. Similar definitions apply to $\prec, \succ$, and $\succcurlyeq$. For two smooth functions, $f(x, t) \leqslant g(x, t)$ is to be understood as the usual order applied in a pointwise sense in both $x$ and $t$ (or just $x$ for $f(x)$ and $g(x)$ ).

We now collect assumptions regarding the boundary and initial-boundary conditions. It is to be understood that in all of the results to follow these assumptions apply. They will not therefore be stated explicitly each time.

(1) (Hypothesis 1) The boundary $\partial \Omega$ is smooth, say $C^{2+\alpha}$, though this condition can be relaxed somewhat. The main requirement is that it satisfies the strong sphere property [29].

(2) (Hypothesis 2) $\left(u_{o}, v_{o}\right) \in \mathbf{C}(\Omega)$ and $u_{o}=v_{o}=0$ on $\partial \Omega$ (compatibility).

For convenience of presentation we shall often use the notation

$$
\begin{aligned}
& f(u, v)=-\omega(u-v)+Q \mathrm{e}^{u}-\alpha u, \\
& g(u, v)=\kappa \omega(u-v)+v(u-v) \mathrm{e}^{u} .
\end{aligned}
$$

To be precise, by blowup we will mean that at least one component of the solution becomes unbounded as $t$ approaches the blowup time, $\tau$ :

$$
\lim _{t \rightarrow \tau} \max _{x \in \bar{\Omega}}(|u|+|v|)=\infty .
$$

Furthermore, we restrict this definition to finite-time blowup, that is $\tau<\infty$.

Finally, we shall often have need of the first eigenfunction and eigenvalue pair, $\left(\phi_{1}, \lambda_{1}\right) \in\left(C^{2}(\Omega) \cap C(\bar{\Omega}), \mathbb{R}\right)$, defined by the problem

$$
-\Delta \phi=\lambda \phi \quad \text { in } \Omega, \quad \phi=0 \quad \text { on } \partial \Omega, \quad \int_{\Omega} \phi(x) \mathrm{d} x=1 .
$$

It is well known that $\phi_{1}$ is positive in $\Omega$ and that $\lambda_{1}>0$.

\section{Local existence}

We begin with the time-dependent problem, for which we first derive conditions for local existence. Conditions for blowup under various limits are established in the following two sections.

The semilinear source terms in Eqs. (2.6) are locally Lipschitz in $u$ and $v$, which guarantees the local existence of solutions in $\mathbf{C}^{1,2}\left(D_{\tau}\right) \cap \mathbf{C}\left(\overline{D_{\tau}}\right)$ when $v \geqslant 0$. In the Appendix we establish a comparison lemma and demonstrate the non-negativity of solutions for $v \geqslant 0$ (Lemma A.1 and Corollary A.2). This comparison lemma is useful for extracting information about the general system (2.6). We also employ it later to reduce the demonstration of blowup (or global existence) to the problem of finding non-global lower (or global upper) solutions, the latter of which also yield estimates 
of the solution profiles. With Lemma A.1 and Corollary A.2 we can now use a continuation argument to demonstrate the local existence of solutions for $v \geqslant 0$.

Lemma 3.1. There exists $\tau \leqslant \infty$ such that a unique solution $\vec{u} \equiv(u, v)$ of (2.6) with $v \geqslant 0$ exists in $[0, \tau) \times \bar{\Omega}$ and $\vec{u} \in \mathbf{C}^{1,2}\left(D_{\tau}\right) \cap \mathbf{C}\left(\overline{D_{\tau}}\right)$. Moreover, $\vec{u}$ exists globally, $\tau=\infty$, or blows up in finite time, $\tau<\infty$.

Proof. For each $n \in \mathbb{N}$, let $y_{n}=\min \{y, n\}$ and $z_{n}=\min \{z, n\}$. We define modified functions which are globally Lipschitz (in $\mathbb{R}^{2}$ ):

$$
\begin{aligned}
& f_{n}(y, z)=-\omega\left(y_{n}-z_{n}\right)+Q \mathrm{e}^{y_{n}}-\alpha y_{n}, \\
& g_{n}(y, z)=\kappa \omega\left(y_{n}-z_{n}\right)+v\left(y_{n}-z_{n}\right) \mathrm{e}^{y_{n}}
\end{aligned}
$$

and consider the modified problem:

$$
\begin{aligned}
& \frac{\partial \hat{u}}{\partial t}-\Delta \hat{u}=f_{n}(\hat{u}, \hat{v}), \\
& \frac{\partial \hat{v}}{\partial t}-\beta \Delta \hat{v}=g_{n}(\hat{u}, \hat{v}), \\
& (\hat{u}, \hat{v})=(0,0), \quad(t, x) \in S_{\tau}
\end{aligned}
$$

with initial conditions

$$
(\hat{u}, \hat{v})=\left(u_{o}, v_{o}\right), \quad x \in \Omega, \quad t=0 .
$$

Note that for $u<n$ and $v<n, f_{n}(u, v)=f(u, v)$ and $g_{n}(u, v)=g(u, v)$. Problem (3.2) has a unique global classical solution, the proof of which can be found in [22,27]. The functions $f_{n}$ and $g_{n}$ are quasi-monotone non-decreasing in $u$ and $v\left(f_{n}(u, v)\right.$ is non-decreasing in $v$ at fixed $u$ and $g_{n}(u, v)$ is non-decreasing in $u$ at fixed $\left.v\right)$. Choose $n \in \mathbb{N}$ such that $u_{o}<n$ and $v_{o}<n$, then the solution of (3.2) is also a solution of (2.6) for as long as $u<n$ and $v<n$. Since $n$ can be chosen arbitrarily, $(\hat{u}, \hat{v})$ is the unique solution of (2.6) as long as it remains bounded. Therefore, unless the unique solution blows up in a finite time $\tau$, it is global.

\section{Blowup for $\alpha=0$ and $v=0$ : no heat loss and no fluid production}

In this section we seek blowup criteria for systems in which no fluid is produced during reaction, $v=0$, and no heat is lost, $\alpha=0$. The criteria will be given as relationships between $\kappa, Q, \beta, \omega$ and the initial conditions $u_{o}$ and $v_{o}$.

We first define two functionals corresponding to the first Fourier coefficients of $u$ and $v$ :

$$
E_{u}(t)=\int_{\Omega} u(x, t) \phi_{1}(x) \mathrm{d} x \quad \text { and } \quad E_{v}(t)=\int_{\Omega} v(x, t) \phi_{1}(x) \mathrm{d} x,
$$

where the eigenpair $\left(\phi_{1}, \lambda_{1}\right)$ is given by (2.10). Integrating Eqs. (2.6) over $\Omega$, applying Green's Theorem and Jensen's inequality yields the following pair of differential inequalities:

$$
\begin{aligned}
\frac{\mathrm{d}}{\mathrm{d} t} E_{u} & =-\lambda_{1} E_{u}-\omega\left(E_{u}-E_{v}\right)+Q \int_{\Omega} \phi_{1} \mathrm{e}^{u} \mathrm{~d} x \\
& \geqslant-\lambda_{1} E_{u}-\omega\left(E_{u}-E_{v}\right)+Q \exp \left(E_{u}\right), \\
\frac{\mathrm{d}}{\mathrm{d} t} E_{v} & =-\lambda_{1} \beta E_{v}+\kappa \omega\left(E_{u}-E_{v}\right),
\end{aligned}
$$

which satisfy initial conditions that correspond to weighted spatial averages of $u_{o}$ and $v_{o}$ over $\Omega$ :

$$
E_{u}(0)=\int_{\Omega} u_{o}(x) \phi_{1}(x) \mathrm{d} x \quad \text { and } \quad E_{v}(0)=\int_{\Omega} v_{o}(x) \phi_{1}(x) \mathrm{d} x .
$$


In order to derive conditions for blowup we compare Eqs. (4.2) with the system of ordinary differential equations (ODE) that is formed by replacing the inequality in (4.2) with equality. As $E_{u} \rightarrow \infty$ or $E_{v} \rightarrow \infty$, condition (2.9) is met [27, Theorem 11.5.1]. The aforementioned ODE system reads as follows:

$$
\begin{aligned}
& \frac{\mathrm{d} U}{\mathrm{~d} t}=-\lambda_{1} U-\omega(U-V)+Q \mathrm{e}^{U}, \\
& \frac{\mathrm{d} V}{\mathrm{~d} t}=-\lambda_{1} \beta V+\kappa \omega(U-V)
\end{aligned}
$$

with initial conditions:

$$
U(0)=E_{u}(0), \quad V(0)=E_{v}(0)
$$

to which there is a unique local-in-time solution, as a consequence of the continuity and local Lipschitz property of the right-hand side, in $\mathbb{R}^{2}$.

To proceed, we examine this system in the $U-V$ phase space. Doing so we discover the existence of a saddle-node bifurcation which separates the space of initial conditions to (4.3) into a region corresponding to global solutions and a region corresponding to unbounded growth in a finite time. The direction of the bifurcation is investigated using normal forms and centre manifold theory (see for example [10]). The main results are given in the next two theorems.

Theorem 4.1. Solutions of system (2.6) blowup for any initial data if the following condition cannot be satisfied for any real and positive $U$ :

$$
Q \mathrm{e}^{U}=\lambda_{1}\left(1+\frac{\omega \beta}{\lambda_{1} \beta+\kappa \omega}\right) U
$$

Proof. Eliminating time as the independent variable in (4.3) we obtain

$$
\frac{\mathrm{d} U}{\mathrm{~d} V}=\frac{-\lambda_{1} U-\omega(U-V)+Q \mathrm{e}^{U}}{-\lambda_{1} \beta V+\kappa \omega(U-V)} .
$$

$\mathrm{d} U / \mathrm{d} V$ is infinite along the line $U=\left(\lambda_{1} \beta+\kappa \omega\right) V / \kappa \omega$ and vanishes when $\lambda_{1} U+\omega(U-V)-Q \mathrm{e}^{U}=0$. For a steady state of (4.3) to exist, these two curves must meet. There are three possibilities: (i) no equilibrium points, (ii) two equilibrium points, (iii) a unique equilibrium point. In general, the nullclines meet at two finite values of $U_{i}$ and $V_{i}$, given by the conditions

$$
Q \mathrm{e}^{U_{i}}-\frac{\lambda_{1} U_{i}}{\lambda_{1} \beta+\kappa \omega}\left(\lambda_{1} \beta+\kappa \omega+\beta \omega\right)=0, \quad V_{i}=\frac{\kappa \omega U_{i}}{\lambda_{1} \beta+\kappa \omega}, \quad i=1,2,
$$

or do not meet at all; case (iii) is special. A sufficient condition for case (i) (no steady states exist) is that the first of Eqs. (4.6) has no positive solutions. This gives condition (4.4). We want to show that under the latter condition all solutions with starting point in the positive quadrant will eventually experience infinite growth in a finite time.

Below the $V$-nullcline $\mathrm{d} U / \mathrm{d} t<0$ and $\mathrm{d} V / \mathrm{d} t>0$, as is easily deduced from Eqs. (4.3), while above this line $\mathrm{d} U / \mathrm{d} t>0$ and $\mathrm{d} V / \mathrm{d} t>0$, to the right of the $U$-nullcline (Fig. 1 is a typical example). To the left of the $U$-nullcline, $\mathrm{d} U / \mathrm{d} t<0$ and $\mathrm{d} V / \mathrm{d} t>0$. Thus, all orbits will eventually satisfy $\mathrm{d} U / \mathrm{d} V>0$, and in particular $\mathrm{d} U / \mathrm{d} t>0$, i.e., both $U$ and $V$ are eventually monotonically increasing; that is, for all $t$ beyond a sufficiently large $t_{*}$. As with the remaining figures, the numerical values that were used will be given in Section 7, where we discuss the numerical results in detail. By uniqueness of solutions and continuation, if $U<\infty$ at $t_{*}$ we have

$$
\frac{\mathrm{d} U}{\mathrm{~d} t}=-\lambda_{1} U-\omega(U-V)+Q \mathrm{e}^{U} \geqslant k U^{2} \text { for some } k>0,
$$

which gives

$$
U \geqslant \frac{k U\left(t_{*}\right)}{k U\left(t_{*}\right)-t} .
$$

Thus, the solution becomes unbounded in finite time (a typical such orbit is shown in Fig. 1). 

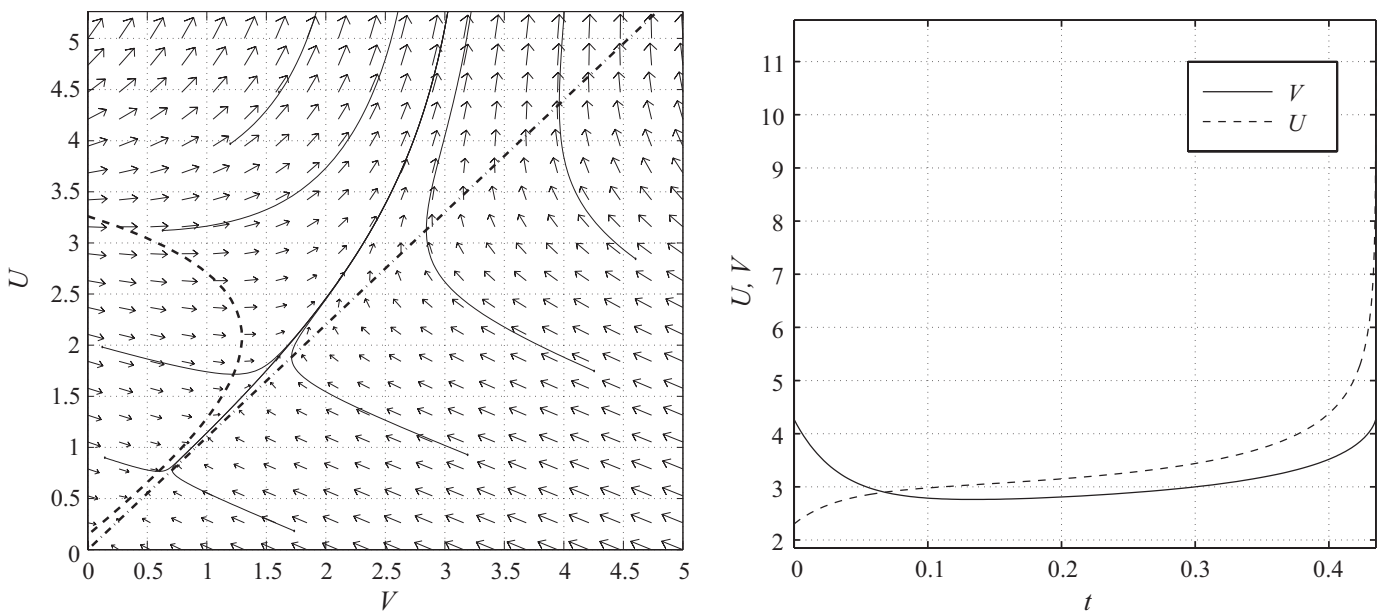

Fig. 1. The left-hand figure is a typical phase plane corresponding to (4.5) when no equilibrium points of (4.3) exist. All orbits eventually experience unbounded growth in a finite time. The dashed-dotted line is the nullcline of $V\left(\lambda_{1} \beta V-\kappa \omega(U-V)=0\right)$ and the dashed curve is the nullcline of $U\left(\lambda_{1} U+\omega(U-V)-Q \mathrm{e}^{U}=0\right)$. The directions of the example orbits can be deduced from the direction of the underlying flow, indicated by the arrows. The right-hand figure is an example solution corresponding to the orbit in the left-hand figure that starts below the $V$-nullcline.
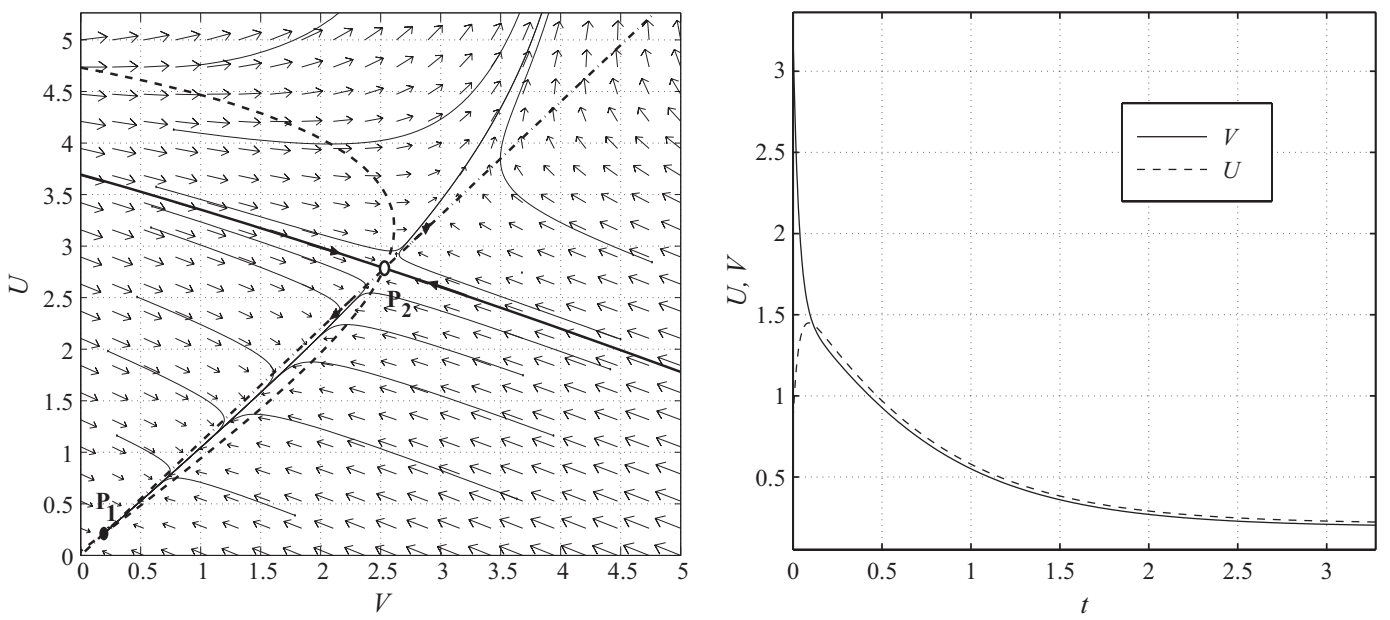

Fig. 2. A typical phase plane corresponding to (4.5) when there are two equilibrium solutions of (4.3). The dashed-dotted line is the nullcline of $V\left(\lambda_{1} \beta V-\kappa \omega(U-V)=0\right)$ and the dashed curve is the nullcline of $U\left(\lambda_{1} U+\omega(U-V)-Q \mathrm{e}^{U}=0\right)$. The solid dark curve is a separatrix corresponding to the stable manifold of the saddle point $\mathrm{P}_{2}$. All orbits with a starting point above the separatrix eventually experience unbounded growth in a finite time. Those starting below the separatrix converge to $\mathrm{P}_{1}$. The directions of the example orbits can be deduced from the direction of the underlying flow, indicated by the arrows. The right-hand figure is an example of a solution converging to $\mathrm{P}_{1}$.

Note that from $\mathrm{d} U / \mathrm{d} t \geqslant-\left(\lambda_{1}+\omega\right) U+Q \mathrm{e}^{U}$, we can obtain the following bound for the blowup time:

$$
\tau \leqslant \int_{U(0)}^{+\infty} \frac{\mathrm{d} z}{-\left(\lambda_{1}+\omega\right) z+Q \mathrm{e}^{z}}<\infty .
$$

When equilibrium points exist, (conditional) stability is possible. To help visualize the analysis that follows, in Fig. 2 we provide an example of $U-V$ phase space when two equilibrium points are present. The dashed and dashed-dotted curves are, respectively, the $U$ and $V$ nullclines of system (4.3), which meet at two distinct points, $\mathrm{P}_{1}\left(U_{1}, V_{1}\right)$ and $\mathrm{P}_{2}\left(U_{2}, V_{2}\right)$. Point $\mathrm{P}_{1}$ is a stable node, while $\mathrm{P}_{2}$ is a saddle. The solid dark curve is a separatrix that is formed by the stable manifold of $\mathrm{P}_{2}$. Orbits that pass through any point below the separatrix converge to point $\mathrm{P}_{1}$, which is therefore an 
asymptotically stable positive attractor, that is $\lim _{t \rightarrow \infty}\|(U, V)\|=\left\|\left(U_{2}, V_{2}\right)\right\|$, where $\|\cdot\|$ can be taken as the Euclidean norm. Orbits with a starting point above the separatrix diverge and correspond to solutions that become unbounded in a finite time. An example of a stable solution is shown in Fig. 2.

The presence of the asymptotically stable positive attractor $P_{1}$ in Fig. 2 ensures that all solutions with a starting point in its basin of attraction (below the separatrix) remain bounded, and blowup cannot be inferred. The saddle-node structure in Fig. 2 arises from bifurcation along the manifold of parameter space (4.7), defined below.

Theorem 4.2. If condition (4.4) can be satisfied, it generally occurs at two positive values of $(U, V), \mathrm{P}_{1}\left(U_{1}, \kappa \omega U_{1} /(\kappa \omega+\right.$ $\left.\left.\beta \lambda_{1}\right)\right)$ and $\mathrm{P}_{2}\left(U_{2}, \kappa \omega U_{2} /\left(\kappa \omega+\beta \lambda_{1}\right)\right)$, where $U_{1}<U_{2}$. A saddle point $\mathrm{P}_{2}$ is created by a saddle-node bifurcation, which may be triggered by parameter variations as follows (with other parameters fixed in each case):

(1) increasing $\lambda_{1}$ (small domains),

(2) decreasing $Q$ (small heat release),

(3) increasing $\omega$ (large heat exchange),

(4) increasing $\beta$ (strong fluid diffusion),

(5) decreasing $\kappa$ (small fluid heat capacity).

Solutions bifurcate along

$$
Q^{*} \mathrm{e}^{U^{*}}=\lambda_{1}^{*}\left(1+\frac{\omega^{*} \beta^{*}}{\lambda_{1}^{*} \beta^{*}+\kappa^{*} \omega^{*}}\right), \quad V^{*}=\frac{\kappa^{*} \beta^{*}}{\lambda_{1}^{*} \beta^{*}+\kappa^{*} \omega^{*}} U^{*}
$$

Solutions of (2.6) blowup if the initial data, $\left(u_{o}, v_{o}\right)$, corresponds to an initial point of problem (4.3) that lies in the positive quadrant of the $U-V$ phase plane between the $U-V$ axes and the separatrix formed by the stable manifold of the saddle point $\mathrm{P}_{2}$ (see Fig. 2 for an example of this structure).

Proof. By the implicit function theorem, the demarcation between existence and non-existence of equilibrium points is the manifold on which the Jacobian corresponding to the right-hand side of (4.3a)-(4.3b) is singular and the nullclines intersect, yielding (4.7). At any point on the manifold (4.7), the linearization of (4.3) reveals that the eigenvalues are

$$
\pi_{1}=0, \quad \pi_{2}=-\frac{\kappa^{*} \omega^{* 2}}{\lambda_{1}^{*} \beta^{*}+\kappa^{*} \omega^{*}}-\left(\lambda_{1}^{*} \beta^{*}+\kappa^{*} \omega^{*}\right),
$$

so that the linearized system has a one-dimensional stable manifold, $L_{\mathrm{S}}$, and a one-dimensional centre manifold, $L_{\mathrm{c}}$. Therefore, system (4.3) has a $C^{\infty}$ one-dimensional local stable invariant manifold and a $C^{m}$ (for any $m$ ) onedimensional local centre invariant manifold, which are, respectively, tangent to $L_{\mathrm{s}}$ and $L_{\mathrm{c}}$ at any point along (4.7) (see [37, Theorem 13.3]). The behaviour of the system and the nature of any bifurcation are determined by the flow in the centre manifold. Consider the behaviour of the system as $\lambda_{1}$ moves through its value on (4.7), with other parameters fixed. Let

$$
U(t)=U^{*}+\mathrm{u}(t), \quad V(t)=V^{*}+\mathrm{v}(t), \quad \lambda_{1}=\lambda_{1}^{*}+\lambda .
$$

Linearizing about a point on (4.7), $\left(V^{*}, U^{*}, Q^{*}, \lambda_{1}^{*}, \beta^{*}, \kappa^{*}, \omega^{*}\right)$ and using the change of variables

$$
\mathrm{y}_{1}=-\frac{\lambda}{\omega^{*}} \frac{\omega^{*} \mathrm{v}+\Lambda \mathrm{u}}{\kappa^{*} \omega^{* 2}+\Lambda^{2}}, \quad \mathrm{y}_{2}=\frac{\Lambda \mathrm{v}-\kappa^{*} \omega^{*} \mathrm{u}}{\kappa^{*} \omega^{* 2}+\Lambda^{2}}
$$

where $\Lambda=\lambda_{1}^{*} \beta^{*}+\kappa^{*} \omega^{*}$ gives

$$
\begin{aligned}
& \frac{\mathrm{dy}}{\mathrm{d} t}=-k_{1}\left(\mathrm{y}_{1}+\mathrm{y}_{2}\right)^{2}+k_{2} \lambda \\
& \frac{\mathrm{dy}}{\mathrm{d} t}=-\left(\kappa^{*} \omega^{* 2} / \Lambda+\omega^{*}\right) \mathrm{y}_{2}-k_{3}\left(\mathrm{y}_{1}+\mathrm{y}_{2}\right)^{2}-k_{4}\left(\beta^{*}-1\right) \lambda
\end{aligned}
$$


and $\mathrm{d} \lambda / \mathrm{d} t=0$, with errors $O\left(\mathrm{y}_{1} \lambda, \mathrm{y}_{2} \lambda, \mathrm{y}_{1}^{3}, \mathrm{y}_{2}^{3}\right)$ and $k_{i}>0, i=1, \ldots, 4$. This system has a centre manifold $\mathrm{y}_{2}=h\left(\mathrm{y}_{1}, \lambda\right)$, $\left|\mathrm{y}_{1}\right|<\delta_{1},|\lambda|<\delta_{2}$, where $\delta_{1}, \delta_{2}>0$. Equating an expansion with another obtained from the chain rule yields the equations for the centre manifold:

$$
\frac{\mathrm{dy}_{1}}{\mathrm{~d} t}=-k_{1} \mathrm{y}_{1}^{2}+k_{2} \lambda+O\left(\lambda \mathrm{y}_{1}, \mathrm{y}_{1}^{3}\right), \quad \frac{\mathrm{d} \lambda}{\mathrm{d} t}=0,
$$

where $k_{1}$ and $k_{2}$ are positive. We see that bifurcation occurs as $\lambda$ passes through zero, i.e., $\lambda_{1}$ passes through $\lambda_{1}^{*}$, from below. Results (2)-(5) are similarly proved.

\section{Global existence and blowup for $\alpha \neq 0$ and $v \neq 0$ : heat loss and fluid production}

In this section we are concerned with behaviour of system (2.6) under the assumption of net fluid production during reaction, which corresponds to $v>0$. We consider two special cases. In the next two subsections we treat adiabatic conditions (no heat is lost), in which case we are able to prove a blowup result, and heat losses $(\alpha>0)$, for which we are able to prove global existence under favourable conditions.

\subsection{Fluid production and no heat loss: $v>0$ and $\alpha=0$}

We employ the comparison Lemma A.1 to look for unbounded lower solutions. This implies the blowup of (2.6), the proof of which can be found in Theorem 11.5.1 of [27]. The main results can summarized as follows:

Theorem 5.1. Assume $u_{o} \geqslant v_{o}$. Solutions of the system (2.6) blowup for any initial data that satisfy $\left(u_{o}, v_{o}\right) \geqslant\left(\psi_{0}, r \psi_{0}\right)$, if the following quantity is positive:

$$
\tau_{o}=\frac{\bar{D}}{a-\lambda_{1}} \ln \left(1+\frac{a-\lambda_{1}}{b E_{0}}\right) .
$$

Moreover, if $\tau_{o}$ is positive the blowup time $\tau$ is bounded above by $\tau_{o} . \psi_{0}$ is defined to be any function that satisfies the problem

$$
-\Delta \psi_{0} \leqslant a \psi_{0}+b \psi_{0}^{2} \quad \text { in } \Omega, \quad \psi=0 \quad \text { on } \partial \Omega,
$$

The quantities $r, \lambda_{1}, a, b, \bar{D}$ and $E_{0}$ are defined by

$$
r= \begin{cases}1-h, h \in(0,1) \text { is arbitrary } & Q>\omega, \\ 1-Q / \omega, & Q<\omega\end{cases}
$$

and

$$
\begin{aligned}
& a=\min \{Q-\omega(1-r),(\kappa \omega+v)(1-r)\} \geqslant 0, \quad b=\min \{Q / 2, v(1-r)\}, \\
& \bar{D}=\max \{1,1 / \beta\}, \quad E_{0}=E(0)=\int_{\Omega} \phi_{1}(x) \psi_{0}(x) \mathrm{d} x .
\end{aligned}
$$

Proof. Let $w(x, t)$ be a non-negative function in $(0, \tau) \times \bar{\Omega}$ that is unbounded in the $L^{\infty}$-norm as $t \rightarrow \tau$, for some set of $\Omega$. Next let $\underline{u}=w$ and $\underline{v}=r w$ be a lower solution of (2.6) for some constant $0<r<1$. In order to satisfy the requirements of a subsolution we need

$$
\left.\begin{array}{l}
\frac{\partial w}{\partial t}-\Delta w \leqslant Q \mathrm{e}^{w}-\omega(1-r) w \\
\frac{\partial w}{\partial t}-\beta \Delta w \leqslant \kappa \omega(1-r) w+v(1-r) w \mathrm{e}^{w}
\end{array}\right\} \quad(t, x) \in D_{\tau},
$$

along with the initial-boundary conditions:

$$
w=0 \quad \text { on }(t, x) \in S_{\tau} \quad \text { and } \quad w(0, x)=w_{0}(x) \leqslant \min \left\{u_{o}, r^{-1} v_{o}(x)\right\} .
$$


Since $\mathrm{e}^{x} \geqslant 1+x+x^{2} / 2, x \in \mathbb{R}_{+}$, we can instead demand that

$$
\left.\begin{array}{l}
\frac{\partial w}{\partial t}-\Delta w \leqslant[Q-\omega(1-r)] w+\frac{Q}{2} w^{2} \\
\frac{\partial w}{\partial t}-\beta \Delta w \leqslant(\kappa \omega+v)(1-r) w+v(1-r) w^{2}
\end{array}\right\} \quad(t, x) \in D_{\tau}
$$

with the quantity $r$ selected as above. This permits us to define a function $\psi(x, t)$ that will satisfy all of the requirements of a subsolution

$$
\begin{aligned}
& \bar{D} \frac{\partial \psi}{\partial t}-\Delta \psi=a \psi+b \psi^{2}, \quad(t, x) \in D_{\tau} \\
& \psi=0 \quad \text { on }(t, x) \in S_{\tau} \quad \text { and } \quad \psi(0, x) \leqslant w_{0}(x)
\end{aligned}
$$

provided that $\psi_{t} \geqslant 0$. For this, a sufficient condition is that $\psi_{0}(x)=\psi(0, x)$ is a lower solution of (5.7), where the latter is defined as follows:

$$
-\Delta \psi_{0} \leqslant a \psi_{0}+b \psi_{0}^{2} \quad \text { in } \Omega, \quad \psi=0 \quad \text { on } \partial \Omega
$$

For a proof of this see Theorem 3.3 in [4]. We now set

$$
E(t)=\int_{\Omega} \phi_{1}(x) \psi(x, t) \mathrm{d} x, \quad E_{0}=E(0)=\int_{\Omega} \phi_{1}(x) \psi_{0}(x) \mathrm{d} x,
$$

where $\left(\phi_{1}, \lambda_{1}\right)$ are defined in (2.10). Following the procedure at the beginning of Section 4 we obtain

$$
E(t)=\frac{\left(a-\lambda_{1}\right) E_{0} \mathrm{e}^{\left(a-\lambda_{1}\right) t / \bar{D}}}{a-\lambda_{1}+b E_{0}\left(1-\mathrm{e}^{\left(a-\lambda_{1}\right) t / \bar{D}}\right)} .
$$

Since $b$ is positive $E$ is guaranteed to become infinite in a finite time $\tau_{o}$, given by (5.1), provided the latter is positive. Therefore, a blowing-up lower solution to (2.6) is given by $(\underline{u}, \underline{v})=(\psi, r \psi)$ with $\left(\underline{u}_{o}, \underline{v}_{o}\right)=\left(\psi_{0}, r \psi_{0}\right)$. This is sufficient to prove the blowup of (2.6) with $\tau \leqslant \tau_{o}$ [27, Theorem 11.5.1].

Note that in the preceding result, the value of $\tau_{o}$ is guaranteed to be positive if $a-\lambda_{1}>0$.

\subsection{Fluid production and heat loss: $v>0$ and $\alpha>0$}

To seek conditions for global existence in the present case, we examine the time evolution of the maximum values of $u$ and $v$ over $\bar{\Omega}$, as $t \rightarrow \infty$. The latter are defined as follows:

$$
M(t)=\max _{x \in \bar{\Omega}} u(x, t), \quad N(t)=\max _{x \in \bar{\Omega}} v(x, t),
$$

where from Lemma A.2 and the boundary conditions we know that both $M$ and $N$ are non-negative. The following Lemma from [11] plays a crucial role:

Lemma 5.2. Let $u \in W^{1,1}((a, b), C(\bar{\Omega}))$ and let there be given for each $t \in(a, b)$ one pair of points $\xi(t), \zeta(t)$ in $\bar{\Omega}$ such that

$$
M(t)=\max _{x \in \bar{\Omega}} u(x, t)=u(t, \xi(t)) \quad \text { and } \quad m(t)=\min _{x \in \bar{\Omega}} u(x, t)=u(t, \zeta(t)),
$$

then the functions $M(t): \mathbb{R} \rightarrow \mathbb{R}$ and $m(t): \mathbb{R} \rightarrow \mathbb{R}$ are almost everywhere differentiable, are in $W^{1,1}((a, b), \mathbb{R})$ and satisfy

$$
M^{\prime}(t)=u_{t}(t, \xi(t)) \quad \text { and } \quad m^{\prime}(t)=u_{t}(t, \zeta(t)) \text { a.e. } t \in(a, b) .
$$

Note that by Lebesgue's dominated convergence theorem, $M$ and $N$ are then absolutely continuous functions on $[a, b]$. For system (2.6), with $\alpha=v=0$, by Corollary A. 2 we know that $m(t)=0$ for both $u$ and $v$, and that $\zeta(t) \in \partial \Omega$. 
Along $x=\xi_{u}(t)$ (subscript refers to the quantity in the case of $u$ or $v$ ), solutions $u$ satisfy $\nabla u=0$ and $\Delta u \leqslant 0$. The same is true of $v$ along $\xi_{v}(t)$. Therefore, using Lemma 5.2, we obtain

$$
\begin{aligned}
M^{\prime}(t)=\partial_{t} u(\xi(t), t) & \leqslant Q \mathrm{e}^{M(t)}-\omega(M(t)-v(x, t))-\alpha M(t) \\
& \leqslant Q \mathrm{e}^{M(t)}-\omega\left(M(t)-v\left(\xi_{v}(t), t\right)\right)-\alpha M(t) \\
& =Q \mathrm{e}^{M(t)}-\omega(M(t)-N(t))-\alpha M(t)
\end{aligned}
$$

and similarly for $v$ :

$$
N^{\prime}(t) \leqslant\left(\kappa \omega+v \mathrm{e}^{M(t)}\right)(M(t)-N(t))
$$

which hold for almost all $t \in(0, \tau)$, for the maximal existence time, $\tau$, of (2.6). The sets $O_{M} \equiv\{t \in(0, \tau): M(t)=0\}$ and $O_{N} \equiv\{t \in(0, \tau): N(t)=0\}$ also satisfy these estimates because of the absolute continuity of $M$ and $N$. Their weak and classical derivatives coincide a.e. $t \in(0, \tau)$. Therefore $M^{\prime}(t)=0$ a.e. $t \in(0, \tau)$ and so (5.13a) holds in the set $O_{M}$. Similarly (5.13b) holds in $O_{N}$ (see [28]).

Now, if we can bound solutions of the system

$$
\begin{aligned}
& M^{\prime}=G(M, N) \equiv Q \mathrm{e}^{M}-\omega(M-N)-\alpha M, \\
& N^{\prime}=H(M, N) \equiv\left(\kappa \omega+v \mathrm{e}^{M}\right)(M-N),
\end{aligned}
$$

then the existence of a global solution is implied.

By analogy with Theorem 4.1 the existence of global solutions to system (5.14) is determined by the existence and nature of its equilibrium points, which are located by the solutions of

$$
Q \mathrm{e}^{M_{*}}=\alpha M_{*}, \quad N_{*}=M_{*} .
$$

There are generally no positive real solutions or two positive real solutions, $M_{*}^{(1)}$ and $M_{*}^{(2)}>M_{*}^{(1)}$. Accordingly, the main result is as follows:

Theorem 5.3. If the condition

$$
Q_{*} \mathrm{e}^{M_{*}}=\alpha_{*} M_{*},
$$

can be satisfied, for some positive $\left(Q_{*}, M_{*}^{(1)}, \alpha_{*}\right)$ and $\left(Q_{*}, M_{*}^{(2)}, \alpha_{*}\right)$, where $M_{*}^{(1)}<M_{*}^{(2)}$, then there exists a neighbourhood $\mathrm{N}$ of $\left(M_{*}^{(1)}, M_{*}^{(1)}\right)$ for which solutions to (2.6) are global if $\left(u_{o}, v_{o}\right) \in \mathrm{N}$. This neighbourhood is contained in the basin of attraction (in the $M-N$ phase space of (5.14)) of the stable node, $\left(M_{*}^{(1)}, M_{*}^{(1)}\right)$, formed by a saddle-node bifurcation along the manifold $Q_{*} \mathrm{e}^{M_{*}}=\alpha_{*}$. More specifically, it is the intersection of the region below the stable manifold of the saddle point, $\left(M_{*}^{(2)}, M_{*}^{(2)}\right)$, and the positive quadrant of the phase space. Bifurcation is triggered by (1) increasing $\alpha$ (greater heat loss) and (2) decreasing $Q$ (lower heat release).

The proof is identical to that of Theorem 4.1 and is therefore omitted. Fig. 3 shows an example of the $M-N$ phase plane in both the absence and presence of equilibrium points.

\section{Analysis of the steady states for $v=\alpha=0$}

To complement the results of Theorems 4.1 and 4.2 of Section 4 we consider steady states of the system (2.6) under the conditions $v=\alpha=0$. Although we are interested here in the special case of $\vec{f}\left(u_{\mathrm{s}}, v_{\mathrm{s}}\right)=\left(Q \mathrm{e}^{u_{\mathrm{s}}}-\omega\left(u_{\mathrm{s}}-v_{\mathrm{s}}\right), \kappa \omega\left(u_{\mathrm{s}}-v_{\mathrm{s}}\right)\right)$, the results obtained are applicable to more general reaction functions $\vec{f}\left(u_{\mathrm{s}}, v_{\mathrm{s}}\right) \in \mathrm{L}^{\text {loc }}\left(\mathbb{R}_{+}\right)$.

We seek functions $u_{\mathrm{s}}(x)$ and $v_{\mathrm{s}}(x)$ satisfying:

$$
\begin{aligned}
& -\Delta u_{\mathrm{s}}=Q \mathrm{e}^{u_{\mathrm{s}}}-\omega\left(u_{\mathrm{s}}-v_{\mathrm{s}}\right), \\
& -\beta \Delta v_{\mathrm{s}}=\kappa \omega\left(u_{\mathrm{s}}-v_{\mathrm{s}}\right)
\end{aligned}
$$



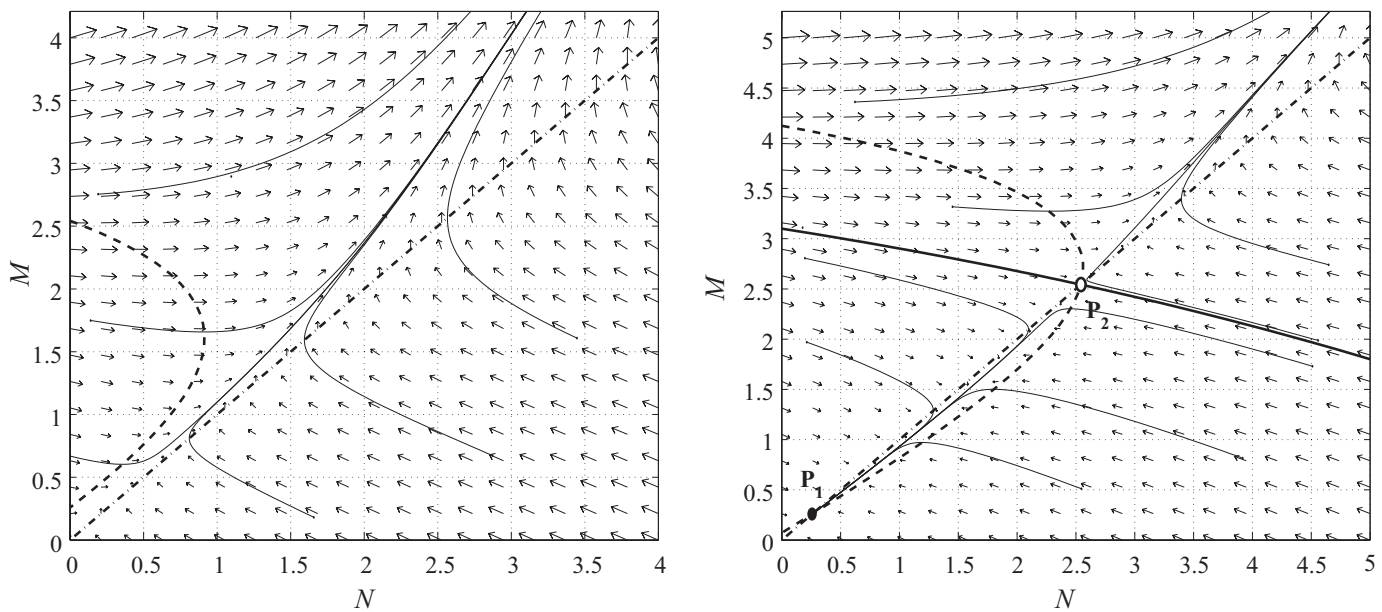

Fig. 3. The left-hand figure is a typical phase plane corresponding to (5.14) when no equilibrium solution exist. The dashed-dotted line corresponds to the nullcline of $N(M-N=0)$ and the dashed curve to the nullcline of $M\left(Q \mathrm{e}^{M}-\omega(M-N)-\alpha M=0\right)$. All examples of orbits, the directions of which are indicated by the arrows, correspond to unbounded growth in a finite time. The right-hand figure is a typical phase plane corresponding to (5.14) when the saddle-node structure exists. The dashed-dotted line corresponds to the nullcline of $N(M-N=0)$ and the dashed curve to the nullcline of $M\left(Q \mathrm{e}^{M}-\omega(M-N)-\alpha M=0\right)$. The dark solid curve is a separatrix formed by the stable manifold of the saddle point, $\mathrm{P}_{2}$. The directions of the example orbits are indicated by the arrows and correspond to unbounded growth for initial conditions above the separatrix and bounded growth for initial conditions below it. The latter terminate at the stable node $\mathrm{P}_{1}$.

subject to the boundary conditions

$$
\left(u_{\mathrm{s}}, v_{\mathrm{s}}\right)=(0,0), \quad x \in \partial \Omega .
$$

Again, we assume that $\partial \Omega$ satisfies Hypothesis 1 of section 2. The definitions of upper and lower solutions of (6.1) are similar to those of (2.6), and are given by (A.1).

\subsection{Existence and uniqueness}

We first show that solutions of (6.1) are non-negative and that $u_{\mathrm{s}} \geqslant v_{\mathrm{s}}$ in $\bar{\Omega}$.

Lemma 6.1. If $u_{\mathrm{s}}, v_{\mathrm{s}}$ are classical solutions of (6.1), then $u_{\mathrm{s}} \geqslant 0$ and $v_{\mathrm{s}} \geqslant 0$ and, moreover, $u_{\mathrm{s}} \geqslant v_{\mathrm{s}}, x \in \bar{\Omega}$.

Proof. Eliminating $\omega\left(u_{\mathrm{s}}-v_{\mathrm{s}}\right)$ in Eqs. (6.1) yields $-\Delta\left(\kappa u_{\mathrm{S}}+\beta v_{\mathrm{S}}\right)>Q \mathrm{e}^{u_{\mathrm{s}}}>0$, so that by the maximum principle, $\kappa u_{\mathrm{s}}+\beta v_{\mathrm{s}} \geqslant 0$. Next suppose that $u_{\mathrm{s}}<0$ at a point $x_{*} \in \bar{\Omega}$. By continuity and the Dirichlet conditions, there must exist a region $R$ in which $u_{\mathrm{s}}<0, v_{\mathrm{s}} \geqslant-\kappa u_{\mathrm{s}} / \beta>0$ and $u_{\mathrm{s}}=0$ on $\partial R$. Therefore

$$
-\Delta u_{\mathrm{s}}=Q \mathrm{e}^{u_{\mathrm{s}}}-\omega u_{\mathrm{s}}+\omega v_{\mathrm{s}}>0, \quad x \in R,
$$

which implies that the minimum of $u_{\mathrm{S}}$ lies on $\partial R$, a contradiction. Next suppose that $v_{\mathrm{s}}<0$ at some point $x_{*} \in \bar{\Omega}$. Arguing in the same fashion as above we reach a contradiction. Thus, both $u_{\mathrm{s}}$ and $v_{\mathrm{s}}$ are non-negative. Finally, suppose that $u_{\mathrm{s}}-v_{\mathrm{s}}<0$ at some point $x_{*} \in \bar{\Omega}$. Then there exists a region $R$ in which $u_{\mathrm{s}}-v_{\mathrm{s}}<0$, and $u_{\mathrm{s}}=v_{\mathrm{s}}$ on $\partial R$. From Eqs. (6.1),

$$
-\Delta u_{\mathrm{s}}=Q \mathrm{e}^{u_{\mathrm{s}}}-\omega\left(u_{\mathrm{s}}-v_{\mathrm{s}}\right)>0, \quad-\beta \Delta v_{\mathrm{s}}=\kappa \omega\left(u_{\mathrm{s}}-v_{\mathrm{s}}\right)<0
$$

so that the minimum of $u_{\mathrm{s}}$, respectively, the maximum of $v_{\mathrm{s}}$, lies on $\partial R$, implying that $u_{\mathrm{s}}-v_{\mathrm{s}} \geqslant 0, x \in \bar{\Omega}$. This contradicts the assumption.

The preceding lemma demonstrates that $\left(u_{\mathrm{s}}, v_{\mathrm{s}}\right)=(0,0)$ is a lower solution of $(6.1)$. The existence of at least one solution can be demonstrated if it is possible to find a bounded sector of the form

$$
\left\langle\underline{\vec{u}_{\mathrm{s}}}, \overline{\overrightarrow{u_{\mathrm{s}}}}\right\rangle \equiv\left\{\vec{u}_{\mathrm{s}}: \underline{\vec{u}_{\mathrm{s}}} \preccurlyeq \vec{u}_{\mathrm{s}} \preccurlyeq \overline{\vec{u}_{\mathrm{s}}}\right\},
$$


where $\vec{u}_{\mathrm{s}} \equiv\left(u_{\mathrm{s}}, v_{\mathrm{s}}\right)$ and the limits of the sector, $\underline{\vec{u}_{\mathrm{s}}}$ and $\overrightarrow{\vec{u}_{\mathrm{s}}}$, are, respectively, lower and upper solutions of (6.1). The latter are said to be ordered if $\overrightarrow{u_{\mathrm{s}}} \preccurlyeq \overrightarrow{\vec{u}_{\mathrm{s}}}$. Given such a sector, existence can be demonstrated using, for example, a monotone-iteration argument [27,31] , or the Leray-Schauder fixed point method [15]. We shall use the former since the uniqueness part of the following proof relies on the monotone sequences [27]. The next result concerns existence and uniqueness of solutions to (6.1):

Theorem 6.2. Given ordered lower and upper solutions of $(6.1), \underline{\vec{u}}_{\mathrm{s}}=\left(\underline{u}_{\mathrm{s}}, \underline{v}_{\mathrm{s}}\right)$ and $\overrightarrow{\vec{u}}_{\mathrm{s}}=\left(\bar{u}_{\mathrm{s}}, \bar{v}_{\mathrm{s}}\right)$, respectively, there exists at least one classical solution of (6.1) in the sector $\left\langle\underline{\vec{u}_{\mathrm{s}}}, \overrightarrow{\vec{u}_{\mathrm{s}}}\right\rangle$. Furthermore, the solutions (if they exist) are unique if $Q-\omega>\lambda_{1}$, where $\lambda_{1}$ is the first eigenvalue of (2.10).

Proof. For the proof of existence and the following notation, the reader is referred to Lemma A.3 in the Appendix. Let $r=u_{\mathrm{s}}^{M}-u_{\mathrm{s}}^{m}$ and $s=v_{\mathrm{s}}^{M}-v_{\mathrm{s}}^{m}$. Since $\left(u_{\mathrm{s}}^{m}, v_{\mathrm{s}}^{m}\right)$ and $\left(u_{\mathrm{s}}^{M}, v_{\mathrm{s}}^{M}\right)$ are solutions of (6.1), using the intermediate value theorem we obtain

$$
\begin{aligned}
-\Delta r=f\left(u_{\mathrm{s}}^{M}, v_{\mathrm{s}}^{M}\right)-f\left(u_{\mathrm{s}}^{m}, v_{\mathrm{s}}^{m}\right) & =r \frac{\partial f}{\partial u_{\mathrm{s}}}\left(\eta_{u_{\mathrm{s}}}, \eta_{v_{\mathrm{s}}}\right)+s \frac{\partial f}{\partial v_{\mathrm{s}}}\left(\eta_{u_{\mathrm{s}}}, \eta_{v_{\mathrm{s}}}\right) \\
& =\left(Q \mathrm{e}^{\left.\eta_{u_{\mathrm{s}}}-\omega\right) r+\omega s,}\right.
\end{aligned}
$$

where $\left(\eta_{u_{\mathrm{s}}}, \eta_{v_{\mathrm{s}}}\right) \in\left\langle\underline{\vec{u}_{\mathrm{s}}}, \overline{\vec{u}_{\mathrm{s}}}\right\rangle$. Multiplication of this equation by $\phi_{1}$, defined in (2.10), integration over $\Omega$ and Green's Theorem then yield

$$
\int_{\Omega}\left(\lambda_{1}-Q \mathrm{e}^{\eta_{u_{\mathrm{s}}}}+\omega\right) r \phi_{1} \mathrm{~d} x=\int_{\Omega} \omega s \phi_{1} \mathrm{~d} x .
$$

Since the right-hand side is non-negative this equation cannot be satisfied if the left-hand side is non-positive, unless $r=s=0$, i.e., $\left(u_{\mathrm{s}}^{m}, v_{\mathrm{s}}^{m}\right)=\left(u_{\mathrm{s}}^{M}, v_{\mathrm{s}}^{M}\right)$. Because any other solution lies between these two solutions (see the last comment in the proof of Lemma A.3), this implies that there is a unique solution when the left-hand is negative. Thus, a sufficient condition for uniqueness is

$$
\sup \left\{\lambda_{1}-Q \mathrm{e}^{\eta_{u_{\mathrm{s}}}}+\omega \mid \eta_{u_{\mathrm{s}}} \in\left(\underline{u}_{\mathrm{s}}, \bar{u}_{\mathrm{s}}\right)\right\} \leqslant 0 \quad \text { satisfied if } Q-\omega>\lambda_{1},
$$

where we have used as a lower solution $\underline{u}_{\mathrm{s}} \equiv 0$.

\subsection{Bounds on existence}

We demonstrate that a bounded sector $\left\langle\vec{u}_{\mathrm{s}}, \overline{\vec{u}_{\mathrm{s}}}\right\rangle$ can be found for small enough values of $Q$, the heat release. We then show that solutions of (6.1) do not exist for $Q>Q^{*}(<\infty)$, where $Q^{*}$ depends on $\omega, \kappa, \beta$ and $\lambda_{1}$, the first eigenvalue of problem (2.10), and that existence depends continuously on $Q$ and $\kappa / \beta$.

Lemma 6.3. Boundary value problem (6.1) possesses at least one solution if there exist positive constants $a$ and $b$ such that

$$
a \geqslant Q \mathrm{e}^{a \psi_{M}} \quad \text { and } \quad \frac{\kappa \omega \psi_{M}}{\beta+\kappa \omega \psi_{M}} \leqslant \frac{b}{a} \leqslant 1
$$

in which $\psi_{M}=\sup _{x \in \Omega} \psi(x)$, where $\psi$ satisfies

$$
-\Delta \psi=1, \quad x \in \Omega, \quad \psi=0, \quad x \in \partial \Omega .
$$

Proof. We look for a bounded upper solution, $\left(\bar{u}_{\mathrm{S}}, \bar{v}_{\mathrm{s}}\right)$, and use as a lower solution $\left(\underline{u}_{\mathrm{s}}, \underline{v}_{\mathrm{s}}\right)=(0,0)$. If we look for an upper solution in the form $\left(\bar{u}_{\mathrm{s}}, \bar{v}_{\mathrm{s}}\right)=(a \psi, b \psi)$, where $\psi$ satisfies (6.9), we require that

$$
\begin{aligned}
& a \geqslant Q \mathrm{e}^{a \psi}-\omega(a-b) \psi, \\
& \beta b \geqslant \kappa \omega(a-b) \psi .
\end{aligned}
$$

Since $\inf _{x \in \Omega} \psi(x)=0$ and $\sup _{x \in \Omega} \psi(x)=\psi_{M}<\infty$, we obtain conditions (6.8). Existence of a solution follows from an application of Theorem 6.2. 
Note that these conditions depend primarily on $\Omega$ (through the function $\psi(x)$ ) and the heat release $Q$.

Lemma 6.4. Non-negative classical solutions to (6.1) do not exist for

$$
Q>Q^{*} \equiv \lambda_{1}\left(1+\frac{\beta \omega}{\lambda_{1} \beta+\kappa \omega}\right)
$$

Proof. Note first that $\mathrm{e}^{u_{\mathrm{s}}} \geqslant 1+u_{\mathrm{s}}$ for $u_{\mathrm{s}} \in \mathbb{R}_{+}$(superlinear growth). Let $\mathbf{D} \equiv-\Delta-\lambda$ with domain in $W_{0}^{2,2}(\Omega)$ and let $\left(\phi_{1}, \lambda_{1}\right)$ be the first eigenpair, normalized so that $\left\|\phi_{1}\right\|_{L^{2}(\Omega)}=1$. Since $\mathbf{D}$ is self-adjoint and by (Sobolev) embedding any classical solution of $(6.1)$ is in $\mathrm{W}_{0}^{2,2}(\Omega)$, we have

$$
\begin{aligned}
0=\left\langle\mathbf{D} \phi_{1}, u_{\mathrm{s}}\right\rangle_{2}=\left\langle\phi_{1}, \mathbf{D} u_{\mathrm{s}}\right\rangle_{2} & =\left\langle\phi_{1}, Q \mathrm{e}^{u_{\mathrm{s}}}-\lambda_{1} u_{\mathrm{s}}-\omega\left(u_{\mathrm{s}}-v_{\mathrm{s}}\right)\right\rangle_{2} \\
& \geqslant\left\langle\phi_{1}, Q-\left(\omega+\lambda_{1}-Q\right) u_{\mathrm{s}}+\omega v_{\mathrm{s}}\right\rangle_{2}, \\
0=\left\langle\mathbf{D} \phi_{1}, v_{\mathrm{s}}\right\rangle_{2}=\left\langle\phi_{1}, \mathbf{D} v_{\mathrm{s}}\right\rangle_{2} & =\left\langle\phi_{1}, \frac{\kappa \omega}{\beta} u_{\mathrm{s}}-\left(\frac{\kappa \omega}{\beta}+\lambda_{1}\right) v_{\mathrm{s}}\right\rangle_{2},
\end{aligned}
$$

where $\langle\cdot, \cdot\rangle_{2}$ is the $L^{2}(\Omega)$ inner product. Eliminating $v_{\mathrm{s}}$ from (6.12a) and (6.12b) yields

$$
\left\langle\phi_{1}, u_{\mathrm{s}}\left(\frac{\kappa \omega}{\beta}-\frac{\Lambda}{\omega}\left(\omega+\lambda_{1}-Q\right)\right)+\frac{Q \Lambda}{\omega}\right\rangle_{2} \leqslant 0
$$

where $\Lambda \equiv \kappa \omega / \beta+\lambda_{1}$. Clearly this condition cannot be satisfied when:

$$
\frac{\kappa \omega}{\beta}-\frac{\Lambda}{\omega}\left(\omega+\lambda_{1}-Q\right) \quad \text { or } \quad Q>Q^{*} \equiv \lambda_{1}\left(1+\frac{\beta \omega}{\lambda_{1} \beta+\kappa \omega}\right) \text {. }
$$

This completes the proof.

Remark 6.5. It is no accident that $Q^{*}$ resembles the right-hand side of condition (4.4), which yields a condition for blowup (if it is not satisfied for any real positive $U$ ). Though we shall not provide the proof, non-existence of a steady state implies blowup of solutions to (2.6) (as is the case for the single temperature equation). Since (4.4) cannot be satisfied for $Q>Q^{*}$, the result of Theorem 4.1 would be reproduced.

Given that there is an upper bound on $Q$, we now ask: given a solution for some $Q>0$, does a (positive) lower bound exist? The answer to this question is to the negative, as demonstrated by the following:

Lemma 6.6. Suppose that classical non-negative solutions to (6.1) exist for some $Q=Q_{*}$ and some $\kappa / \beta=\kappa_{*} / \beta_{*}$. Then classical non-negative solutions to (6.1) exist for all $Q \in\left[0, Q_{*}\right]$ and $\kappa / \beta \in\left[0, \kappa_{*} / \beta_{*}\right]$.

Proof. Let $\left(u_{\mathrm{s}}^{*}, v_{\mathrm{s}}^{*}\right)$ be a solution of (6.1) with $Q=Q_{*}$, then for any $Q \in\left[0, Q_{*}\right]$, we have

$$
\begin{aligned}
& \Delta u_{\mathrm{s}}^{*}+Q \mathrm{e}^{u_{\mathrm{s}}^{*}}-\omega\left(u_{\mathrm{s}}^{*}-v_{\mathrm{s}}^{*}\right) \leqslant 0, \\
& -\Delta v_{\mathrm{s}}^{*}=\frac{\kappa}{\beta} \omega\left(u_{\mathrm{s}}^{*}-v_{\mathrm{s}}^{*}\right), \\
& \left(u, v_{\mathrm{s}}\right)=(0,0), \quad x \in \partial \Omega .
\end{aligned}
$$

Thus $\left(u_{\mathrm{s}}^{*}, v_{\mathrm{s}}^{*}\right)$ is an upper solution for (6.1) with $Q \in\left[0, Q_{*}\right]$, so that solutions exist in this entire range. Likewise, if $\left(u_{\mathrm{s}}^{*}, v_{\mathrm{s}}^{*}\right)$ is a solution of (6.1) with $\kappa_{*}$ and $\beta_{*}$, then for any $\kappa$ and $\beta$ such that $0 \leqslant \kappa / \beta \leqslant \kappa_{*} / \beta_{*},\left(u_{\mathrm{s}}^{*}, v_{\mathrm{s}}^{*}\right)$ is an upper solution of (6.1) and the result follows.

We next present a result that provides a link between the system (2.6) and its steady states (6.1):

Corollary 6.7. The following global existence and blowup alternatives for system (2.6) hold: if condition (6.8) can be satisfied for real and positive $a$ and $b$, solutions are global for any initial data $\left(u_{o}, v_{o}\right)$. If $Q-\omega>\lambda_{1}$, the solution 
converges to the unique solution of (6.1), otherwise it converges to the maximal or minimal solution of (6.1), depending on the initial data.

Proof. See Theorem 6.2 above and Theorems 10.5.1 and 10.5.3 of [27].

\section{Summary and discussion}

To facilitate a discussion of the results contained in the previous sections it is useful at this point to draw a comparison with the results from a single-temperature model (2.4).

\subsection{Single-temperature results}

Assuming the expansion $T=1+u / E_{\mathrm{a}}, \psi=1-\phi_{0}+w / E_{\mathrm{a}}$, where $u=v$ is the single temperature of the system, Eq. (2.4) can be written as follows:

$$
\begin{aligned}
& \frac{\partial u}{\partial t}=D \Delta u+Q \mathrm{e}^{u}-\alpha u, \quad(t, x) \in D_{\tau}, \\
& u=0, \quad(t, x) \in S_{\tau}, \quad u(x, 0)=u_{o}(x),
\end{aligned}
$$

for the maximal existence time, $\tau$, and where

$$
D=\frac{1-\phi_{0}+\gamma \phi_{0}}{1-\phi_{0}}, \quad \alpha=\frac{\tilde{\alpha}}{1-\psi_{0}} .
$$

When $\alpha=\widetilde{v}=0$ we can apply the procedure at the beginning of Section 4 to obtain

$$
\frac{\mathrm{d} E}{\mathrm{~d} t} \geqslant-D \lambda_{1}+Q \mathrm{e}^{E}
$$

where

$$
E(t)=\int_{\Omega} \phi_{1} u(x, t) \mathrm{d} x, \quad E(0)=\int_{\Omega} \phi_{1} u_{o}(x) \mathrm{d} x .
$$

Non-existence of fixed points of (7.3) implies that solutions, $E(t)$, are unbounded for any positive $E(0)$ and this in turn implies that solutions to (7.1) become unbounded in a finite time. Fixed points, if they exist, satisfy the algebraic equation:

$$
\lambda_{1} E\left(\frac{1-\phi_{0}+\gamma \phi_{0}}{1-\phi_{0}}\right)=Q \mathrm{e}^{E}
$$

of which there are generally two real positive solutions, $E_{1}$ and $E_{2}$. If $E(0) \geqslant E_{2}$ solutions blowup [20]. We compare (7.5) with condition (4.4), or equivalently condition (4.7), to better understand the effect of the heat exchange between the two phases.

(1) Firstly, in the limit $\omega \rightarrow \infty$, condition (4.4) becomes

$$
\lambda_{1} U_{i}\left(\frac{1-\phi_{0}+\gamma \phi_{0}}{1-\phi_{0}}\right)=Q \mathrm{e}^{U_{i}}, \quad V_{i}=U_{i}, \quad i=1,2
$$

having used the definitions (2.7). This is precisely condition (7.5) so problem (2.6) is consistent, in the limit $\omega \rightarrow \infty$, with the single-temperature model.

(2) For a finite $\omega$, we can re-write condition (4.4), using the (2.7), as

$$
\begin{aligned}
Q \mathrm{e}^{U_{i}} & =\frac{\lambda_{1} U_{i}}{1-\phi_{0}}\left(1-\phi_{0}+\gamma \phi_{0}-\frac{\gamma^{2} \phi_{0}^{2} \lambda_{1}}{\gamma \phi_{0} \lambda_{1}+\omega\left(1-\phi_{0}\right)}\right) \\
& =\lambda_{1} U_{i}\left(\frac{1-\phi_{0}+\gamma \phi_{0}}{1-\phi_{0}}-G\right),
\end{aligned}
$$


where

$$
\begin{aligned}
G & \equiv \frac{\gamma^{2} \phi_{0}^{2} \lambda_{1}}{\left(\gamma \phi_{0} \lambda_{1}+\omega\left(1-\phi_{0}\right)\right)\left(1-\phi_{0}\right)}, \\
V_{i} & =\frac{\kappa \omega U_{i}}{\lambda_{1} \beta+\kappa \omega}, \quad i=1,2 .
\end{aligned}
$$

The difference between the conditions (4.4) and (7.5) is therefore contained in the constant $G>0$. The ultimate effect of $G$ is to broaden the range of $Q$ and $\phi_{0}$ for which unconditional blowup (for any initial condition) is achieved. Recall that when two fixed points exist (one saddle point and one stable node) we can only claim that blowup will occur for initial conditions of (2.6) whose weighted spatial average corresponds to initial conditions of (4.3) outside the basin of attraction of the stable node. Additionally, there is an effect on the initial conditions required for blow up, through the relationship between $u_{o}$ and $v_{o}$, but this is not generally easy to extract.

(3) Notice also that condition (6.11) is consistent with the single-temperature result in the limit $\omega \rightarrow \infty$. If we were to apply the procedure of Lemma 6.4 to steady-state solutions of (7.1) we would obtain the bound $Q<\lambda_{1} D$, which, using definitions (2.7), is the same as (6.11) in the limit $\omega \rightarrow \infty$. For a finite $\omega,(6.11)$ can be written as $Q>\lambda_{1}(D-G)$, where $G$ is defined above, a result which is implied by (7.7).

Remark 7.1. We point out that there are stronger results pertaining to system (7.1), which can be found in [20,30], using a more sophisticated Fourier coefficient method. For example, if two solutions of the corresponding steady state problem exist, the maximal solution acts as a critical initial condition.

Consider now $v \neq 0$ and $\alpha \neq 0$. Assuming that $u \in W^{1,1}((a, b), C(\bar{\Omega}))$ we can apply the procedure at the beginning of Section 5.2 to obtain the following differential inequality:

$$
R^{\prime}(t) \leqslant Q \mathrm{e}^{R(t)}-\alpha R(t) \quad \text { where } R(t)=\max _{x \in \bar{\Omega}} u(x, t)
$$

and we have used Lemma 5.2, that is, there is a family of points $\xi(t) \in \bar{\Omega}$ such that: $R(t)=\max _{x \in \bar{\Omega}} u(x, t)=u(t, \xi(t))$ and $R^{\prime}(t)=u_{t}(t, \xi(t))$ a.e. $t \in(a, b)$. This equation implies that solutions to (6.1) are global if there exist solutions, $y$, to $Q \mathrm{e}^{y}-\alpha y$, which is precisely condition (5.16) of Theorem 5.3. The difference between the global existence conditions is therefore contained entirely in the initial data, $\left(u_{o}, v_{o}\right)$. As an example of the effect on the latter we examine the eigenvalues of the linearization about the saddle point $\left(M_{*}^{(2)}, M_{*}^{(2)}\right)$, defined in (5.15), in the limit $\kappa \rightarrow \infty$. They have the asymptotic form

$$
\pi_{1}=-\alpha\left(1-M_{*}^{(2)}\right)+o(1) \quad \text { and } \quad \pi_{2}=-\kappa \omega-\left(v \mathrm{e}^{M_{*}^{(2)}}+\omega\right)+o(1) .
$$

This yields the eigenvectors $\vec{e}_{1} \sim(1,1)$ and $\vec{e}_{2} \sim(1,-1 / \kappa)$, from which we can approximate the stable manifold corresponding to the saddle-point as

$$
\kappa\left(M-M_{*}^{(2)}\right)=M_{*}^{(2)}-N, \quad \kappa \rightarrow \infty .
$$

Recall that this is the boundary between unbounded growth and convergence of the comparison system (5.14), i.e., we can approximate the range of initial conditions for which the system has a global solution by those for which

$$
N(0)+\kappa M(0)<(1+\kappa) M_{*}^{(2)} \text { as } \kappa \rightarrow \infty .
$$

\subsection{Dual-temperature results}

(1) $v=\alpha=0$. As remarked above, the dual-temperature effects enter through the constant $G$ in Eq. (7.7) when $\omega$ is finite. Analysis of the steady states (if they exist) reveals a stable node $\left(U_{1}, V_{1}\right)$ and a saddle point $\left(U_{2}, V_{2}\right)$ at the lower and higher valued solutions of problem (4.3). Unsurprisingly, the presence of a cooler fluid component in the pores of a reactive solid material has no effect if there is no heat exchange between the solid matrix and this 
fluid $(\omega=0)$; for finite $\omega$ there is heat exchange and the most drastic effect is when $\omega \rightarrow \infty$, in which case $G \rightarrow 0$ and the cooling of the solid (by thermal diffusion) is enhanced by thermal diffusion in the fluid via the term $\gamma \phi_{0}$ on the right-hand side of (7.7). For finite $\omega$, this cooling effect is reduced as $G$ increases.

The effect of cooling is to increase the range of $Q$ and $\phi_{0}$ for which a steady attracting state $\left(U_{1}, V_{1}\right)$ can exist, and hence to reduce the possibility of blowup. This effect is demonstrated in the numerical examples displayed in Figs. 1-3 (see below).

(2) $v \neq 0, \alpha \neq 0$. In the case $v \neq 0$, the transfer of heat from the solid to the fluid phase represented by $\omega$ is augmented by the reaction product. In the case where the fluid phase is a gas, this contribution is likely to be dominant unless $v$ is $O(1)$, i.e., $\widetilde{v}=O\left(\delta E_{\mathrm{a}}\right)$, where $\delta \sim 10^{-3}$, or is very large, perhaps achieved by forced convection by a high-speed gas flow through the porous medium.

If the fluid phase is liquid, with $\delta \sim 1$, these restrictions are much less stringent; the numerical examples are more representative of a solid/liquid scenario.

\subsubsection{Numerical examples}

The numerical examples demonstrated in Figs. 1 and 2 are computed for values $\lambda_{1}=1, \beta=2, \omega=\kappa=5, v=\alpha=0$ and $Q=0.8$ (Fig. 1) or $Q=0.25$ (Fig. 2). These figures correspond to, roughly, numerical values of $E_{\mathrm{a}}^{\prime}=8 \times 10^{4} \mathrm{~J} \mathrm{~mol}^{-1}$, $A^{\prime}=10^{5} \mathrm{~s}^{-1}, Q^{\prime}=10^{5} \mathrm{~J} \mathrm{~kg}^{-1}$ and $T_{\mathrm{a}}^{\prime}=500 \mathrm{~K}$, together with $\gamma, \sigma$ and $\delta$ all $\approx 1$, and the heat-exchange coefficient $\omega^{\prime} \approx 10^{3} \mathrm{~J} \mathrm{~mol}^{-1} \mathrm{~s}^{-1}$. This latter quantity, and hence $\omega$, is likely to vary very widely with the physical properties of the materials, especially tortuosity and porosity, particle size and packing for the case of powders and vigour of fluid motion, but the form of the Eqs. (4.3) enable us to readily envisage the range of possibilities for $0<\omega<\infty$.

Fig. 3 illustrates similarly a typical scenario for $v \neq 0$. In this case the values used were $\omega=2, v=1, \alpha=1$, and $\kappa=2$, together with and $Q=0.6$ (left-hand figure) and $Q=0.2$ (right-hand figure). The corresponding physical and chemical properties are as in the case with $v=\alpha=0$.

Note that $v=O(1)$ implies that

$$
\frac{\widetilde{v}\left(1-\phi_{0}\right)}{E_{\mathrm{a}} \delta \phi_{0}}=O(1)
$$

In the case of a gas, this can be if $\widetilde{v}=O\left(\delta E_{\mathrm{a}}\right)$, which for typical values of $\delta$ and $E_{\mathrm{a}}$ means that $\widetilde{v}<1 \%$.

In the case of a liquid, we have $\delta \sim 1$, and hence the term $\widetilde{v}$ is formally of $O\left(E_{\mathrm{a}}^{-1}\right)$ and therefore negligible in the expansions. However, for the values above, the ratio of the first to the second term on the right-hand side of equation (2.1b) is $\widetilde{v} \rho_{\mathrm{s}}^{\prime} C_{\mathrm{s}}^{\prime} A^{\prime} \mathrm{e}^{E_{\mathrm{a}}} / \omega^{\prime}$. If $\rho_{\mathrm{s}}^{\prime}=660 \mathrm{Kg} \mathrm{m}^{-3}$ and $C_{\mathrm{s}}^{\prime}=750 \mathrm{~J} \mathrm{Kg}^{-1} \mathrm{~K}^{-3}$, this is approximately $10^{2} \widetilde{v} / \omega^{\prime}$, which may not be negligible unless $\omega^{\prime}$ is large. But the balance is, unsurprisingly, highly sensitive to the value of $E_{\mathrm{a}}^{\prime}$ through the Arrhenius exponential term.

Direct numerical comparison of the two cases, i.e., $v=0$ and $v \neq 0$, is difficult because the variables $M$ and $N$ relate to the maxima of $u(x, t)$ and $v(x, t)$ in the domain, whilst the variables $U$ and $V$ are averages over the domain. Nevertheless, the effects on blowup behaviour of taking into account the differing temperatures of the solid and fluid components are broadly similar in each case.

\section{Appendix A}

\section{A.1. Comparison lemma and non-negativity (time-dependent problem)}

Lemma A.1. For $t<\tau$, let $(\bar{u}, \bar{v})$ satisfy:

$$
\begin{aligned}
& \frac{\partial \bar{u}}{\partial t}-\Delta \bar{u} \geqslant f(\bar{u}, \bar{v}), \\
& \frac{\partial \bar{v}}{\partial t}-\beta \Delta \bar{v} \geqslant g(\bar{u}, \bar{v}), \\
& (\bar{u}, \bar{v}) \succcurlyeq(0,0), \quad(t, x) \in S_{\tau}, \\
& (\bar{u}, \bar{v}) \succcurlyeq\left(u_{o}, v_{o}\right), \quad x \in \Omega, \quad t=0 .
\end{aligned}
$$


where $(\bar{u}, \bar{v})$ is called an upper solution of (2.6) and a lower solution, $(\underline{u}, \underline{v})$, is defined by reversed inequalities in (A.1). We have the following possibilities:

(1) If $v=0$, solutions of $(2.6)$ satisfy $(\underline{u}, \underline{v}) \preccurlyeq(u, v) \preccurlyeq(\bar{u}, \bar{v})$ in $D_{\tau} \cup \Gamma_{\tau}$.

(2) If $v>0$, solutions of (2.6) satisfy $(\underline{u}, \underline{v}) \preccurlyeq(u, v) \preccurlyeq(\bar{u}, \bar{v})$ in $D_{\tau} \cup \Gamma_{\tau}$ provided that $u_{o} \geqslant v_{o}$ and $Q>\alpha$.

(3) If $v<0$, solutions of (2.6) satisfy $(\underline{u}, \underline{v}) \preccurlyeq(u, v) \preccurlyeq(\bar{u}, \bar{v})$ in $D_{\tau} \cup \Gamma_{\tau}$ provided that $u_{o} \geqslant v_{o}, Q>\alpha$ and $\kappa \omega+v<0$.

Proof. Firstly, assume $v \geqslant 0$ and let $\xi=\bar{u}-u$ and $\eta=\bar{v}-v$. By continuity, if either or both of $\xi$ and $\eta$ become negative there exists

$$
t^{*}=\sup \{t: \xi \geqslant 0 \text { and } / \text { or } \eta \geqslant 0\}<\tau .
$$

Suppose $\xi=0$ at $t^{*}$, for some $x^{*} \in \Omega$ and $\eta>0$. Note that $x^{*}$ does not lie on $\Gamma_{\tau}$ because of the boundary conditions. By construction, and by continuity, $\xi_{t}\left(t^{*}, x^{*}\right)<0, \nabla \xi\left(t^{*}, x^{*}\right)=0$, and $\Delta \xi\left(t^{*}, x^{*}\right) \geqslant 0$. From Eqs. (2.6a) and (A.1a) we then obtain

$$
\frac{\partial \xi}{\partial t}-\Delta \xi \geqslant f(\bar{u}, \bar{v})-f(u, v)=Q\left(\mathrm{e}^{\bar{u}}-\mathrm{e}^{u}\right)-\omega(\xi-\eta)-\alpha \xi=\omega \eta>0
$$

at $\left(t^{*}, x^{*}\right)$, noting that by assumption $\eta\left(t^{*}, x^{*}\right)>0$. As a result $\xi_{t}\left(t^{*}, x^{*}\right)>0$, a contradiction. If we instead assume that $\eta\left(t^{*}, x^{*}\right)=0$ and $\xi>0$ in $D_{t^{*}}$, we obtain the inequality

$$
\frac{\partial \eta}{\partial t}-\beta \Delta \eta \geqslant g(\bar{u}, \bar{v})-g(u, v)=\kappa \omega(\xi-\eta)+v \mathrm{e}^{\bar{u}}(\bar{u}-\bar{v})+v \mathrm{e}^{u}(u-v)
$$

at $\left(t^{*}, x^{*}\right)$. We now consider two possibilities.

- $v=0$ : In this case $\eta_{t}\left(t^{*}, x^{*}\right)>0$ because $\Delta \eta\left(t^{*}, x^{*}\right) \geqslant 0$ and $\xi\left(t^{*}, x^{*}\right)>0$. This is again a contradiction. The only other possibility is that $\xi=\eta=0$ at $\left(t^{*}, x^{*}\right)$. But then Eqs. (A.2) and (A.3) yield

$$
\left.\begin{array}{l}
\frac{\partial \xi}{\partial t}-\Delta \xi \geqslant 0 \\
\frac{\partial \eta}{\partial t}-\beta \Delta \eta \geqslant 0
\end{array}\right\} \quad(t, x)=\left(t^{*}, x^{*}\right)
$$

and since $\Delta \xi \geqslant 0$ and $\Delta \eta \geqslant 0$, we obtain $\xi_{t} \geqslant 0$ and $\eta_{t} \geqslant 0$. This is again a contradiction.

- $v \geqslant 0$ : Here we use the hypothesis $u_{o} \geqslant v_{o}$ to obtain $u-v \geqslant 0$ in $D_{\tau} \cup \Gamma_{\tau}$. To see this, we define $\left(x_{+}, t_{+}\right)$by $t_{+}=\sup \{t: u-v \geqslant 0\}<\tau$ at $x=x_{+}$. We can then define $\delta$ such that for each $t \in\left(t_{+}, t_{+}+\delta\right]$ there is a region $J_{t} \subset \subset \Omega$ in which $u<v$ and $u=v$ on $\partial J_{t}$. Furthermore, $u=v$ on $J_{t_{+}}=\left\{x_{+}\right\}$. From Eqs. we then find that

$$
\left.\begin{array}{l}
\frac{\partial u}{\partial t}-\Delta u>0 \\
\frac{\partial v}{\partial t}-\beta \Delta v<0
\end{array}\right\} \quad(t, x) \in \bigcup_{t \in\left(t_{+}, t_{+}+\delta\right]} J_{t}
$$

if $Q>\alpha$. These equations and the maximum principle imply that $u(v)$ attains its minimum (maximum) on $J_{t_{+}} \cup \bigcup_{t \in\left(t_{+}, t_{+}+\delta\right]} \partial J_{t}$, where by definition $u=v$. Therefore $u>v$, a contradiction. Now using Eq. (A.3) we see that $\partial \eta / \partial t>0$ at a point where $\eta=0$ which is a contradiction. Applying the argument above for $\xi=\eta=0$ at $\left(t^{*}, x^{*}\right)$ in the case of $v=0$ also gives a contradiction for $v>0$. Thus, we have shown that $\xi$ and $\eta$ are non-negative. Applying precisely the same reasoning with $\xi=u-\underline{u}$ and $\eta=v-\underline{v}$ shows again that $\xi$ and $\eta$ are non-negative.

This completes the proof of (1) and (2). For part (3) we again assume that $u_{o} \geqslant v_{o}$. If $u \leqslant v$ there exists $\left(x_{-}, t_{-}\right), x_{-} \in \Omega$, at which $u=v$. Then, we find that

$$
\left.\begin{array}{l}
\frac{\partial u}{\partial t}-\Delta u=Q \mathrm{e}^{u}-\omega(u-v)-\alpha u>0 \\
\frac{\partial v}{\partial t}-\beta \Delta v=(u-v)\left(\kappa \omega+v \mathrm{e}^{u}\right) \leqslant 0
\end{array}\right\} \quad(t, x) \in D_{t_{-}}
$$


if $Q>\alpha$ and $\kappa \omega+v>0$. By the maximum principle, the minimum of $u$ (maximum of $v$ ) is attained on the parabolic boundary $\Gamma_{t_{-}}$. But this contradicts $u=v$ at $\left(x_{-}, t_{-}\right)$. The required proof is therefore complete.

Corollary A.2. For $v \geqslant 0$, any solution $\vec{u} \equiv(u, v) \in \mathbf{C}^{1,2}\left(D_{\tau}\right) \cap \mathbf{C}\left(\overline{D_{\tau}}\right)$ of $(2.6)$ is non-negative in $\overline{D_{\tau}}$.

Proof. For $v=0$ we apply the comparison lemma above using $(0,0)$ as a lower solution. For $v>0$ we do not need the requirement that $u_{o} \geqslant v_{o}$ to obtain non-negativity. Define $t^{*}=\sup \{t: u \geqslant 0$ or $v \geqslant 0\}$ and suppose $u=0$ at $t^{*}$, for some $x^{*} \in \Omega$. By construction, $u_{t}\left(t^{*}, x^{*}\right)<0$ and $\Delta u\left(t^{*}, x^{*}\right) \geqslant 0$. Using Eq. (2.6a) we obtain

$$
\frac{\partial u}{\partial t}-\Delta u=Q+\omega v>0
$$

which is a contradiction. For $v=0$ at $\left(t^{*}, x^{*}\right)$ Eq. (2.6b) yields

$$
\frac{\partial v}{\partial t}-\beta \Delta v=\kappa \omega u+v u \mathrm{e}^{u}>0
$$

a contradiction. When $v=u=0$ at $\left(t^{*}, x^{*}\right)$ we reach a contradiction in a similar fashion.

\section{A.2. Existence (steady state)}

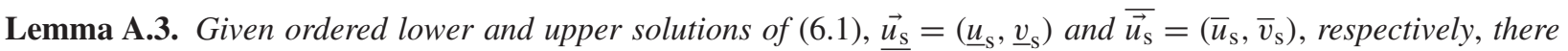
exists at least one classical solution of $(6.1)$ in the sector $\left\langle\underline{\vec{u}_{\mathrm{s}}}, \overline{\overrightarrow{u_{\mathrm{s}}}}\right\rangle$.

Proof. Let $\left(\bar{u}_{\mathrm{s}_{0}}, \bar{v}_{\mathrm{s}_{0}}\right)=\left(\bar{u}_{\mathrm{s}}, \bar{v}_{\mathrm{s}}\right)$ and $\left(\underline{u}_{\mathrm{s}_{0}}, \underline{v}_{\mathrm{s}_{0}}\right)=\left(\underline{u}_{\mathrm{s}}, \underline{v}_{\mathrm{s}}\right)$ and define the sequence $\left(\bar{u}_{\mathrm{s}_{i}}, \bar{v}_{\mathrm{s}_{i}}\right)_{i=1}^{\infty}$ by

$$
\begin{aligned}
& -\Delta \bar{u}_{\mathrm{s}_{i}}+\omega \bar{u}_{\mathrm{s}_{i}}=Q \mathrm{e}^{\bar{u}_{\mathrm{s}_{i-1}}}+\omega \bar{v}_{\mathrm{s}_{i-1}} \equiv F\left(\bar{u}_{\mathrm{s}_{i-1}}, \bar{v}_{\mathrm{s}_{i-1}}\right), \\
& -\beta \Delta \bar{v}_{\mathrm{s}_{i}}+\kappa \omega \bar{v}_{\mathrm{s}_{i-1}}=\kappa \omega \bar{u}_{\mathrm{s}_{i-1}} \equiv G\left(\bar{u}_{\mathrm{s}_{i-1}}, \bar{v}_{\mathrm{s}_{i-1}}\right), \\
& \left(\bar{u}_{\mathrm{s}_{i}}, \bar{v}_{\mathrm{s}_{i}}\right)=(0,0), \quad x \in \partial \Omega,
\end{aligned}
$$

where $F\left(u_{\mathrm{s}}, v_{\mathrm{s}}\right)(x) \equiv\left(f\left(u_{\mathrm{s}}\right)-\omega\left(u_{\mathrm{s}}-v_{\mathrm{s}}\right)\right)(x)$ and $G\left(u_{\mathrm{s}}, v_{\mathrm{S}}\right)(x) \equiv \kappa \omega\left(u_{\mathrm{s}}-v_{\mathrm{s}}\right)(x)$ are Nemetskyii operators. The sequence $\left(\underline{u}_{\mathrm{S}_{i}}, \underline{v}_{\mathrm{S}_{i}}\right)_{i=1}^{\infty}$ has an identical definition but with $\left(\underline{u}_{\mathrm{S}_{0}}, \underline{v}_{\mathrm{s}_{0}}\right)$ as the initial iteration. The Lipschitz property of $f$ and $g$ ensures that $F$ and $G$ are in $C^{\alpha}(\bar{\Omega})$, for $\left(\bar{u}_{\mathrm{S}}, \bar{v}_{\mathrm{S}}\right)$ and $\left(\underline{u}_{\mathrm{S}}, \underline{v}_{\mathrm{S}}\right)$ in $\mathbf{C}^{\alpha}(\bar{\Omega})$. Thus, from the Schauder estimates:

$$
\left|\vec{u}_{\mathrm{s}}\right|_{\mathbf{C}^{2+\alpha}(\bar{\Omega})} \leqslant C|(F(x), G(x))|_{\mathbf{C}^{\alpha}(\bar{\Omega})}, \quad C>0,
$$

where $\left|\left(\psi_{1}, \psi_{2}\right)\right|_{\mathbf{C}^{m+\alpha}(\bar{\Omega})}=\left|\psi_{1}\right|_{C^{m+\alpha}(\bar{\Omega})}+\left|\psi_{2}\right|_{C^{m+\alpha}(\bar{\Omega})}, m \in \mathbb{N}$, both sequences are well defined (the solutions to (A.9) are classical). By application of the maximum principle and the definition of upper and lower solutions, these sequences satisfy the monotone property:

$$
\underline{u}_{\mathrm{s}} \leqslant \underline{u}_{\mathrm{s}_{i}} \leqslant \underline{u}_{\mathrm{s}_{i+1}} \leqslant \bar{u}_{\mathrm{s}_{i+1}} \leqslant \bar{u}_{\mathrm{s}_{i}} \leqslant \bar{u}_{\mathrm{s}} \quad \forall i \in \mathbb{N} .
$$

For example, let $w_{1}=\bar{u}_{\mathrm{s}}-\bar{u}_{\mathrm{s}_{1}}$ and $z_{1}=\bar{v}_{\mathrm{s}}-\bar{v}_{\mathrm{s}_{1}}$, then

$$
\begin{aligned}
& -\Delta w_{1}+\omega w_{1} \geqslant Q \mathrm{e}^{\bar{u}_{\mathrm{s}}}+\omega \bar{v}_{\mathrm{s}}-\left(Q \mathrm{e}^{\bar{u}_{\mathrm{s}}}+\omega \bar{v}_{\mathrm{s}}\right)=0, \\
& -\beta \Delta z_{1}+\kappa \omega z_{1} \geqslant \kappa \omega \bar{u}_{\mathrm{s}}-\kappa \omega \bar{u}_{\mathrm{s}_{1}}=0, \\
& \left(w_{1}, z_{1}\right) \succcurlyeq(0,0), \quad x \in \partial \Omega
\end{aligned}
$$

yielding $\left(w_{1}, z_{1}\right) \succcurlyeq(0,0)$ or $\left(\bar{u}_{\mathrm{s}_{1}}, \bar{v}_{\mathrm{s}_{1}}\right) \preccurlyeq\left(\bar{u}_{\mathrm{s}}, \bar{v}_{\mathrm{s}}\right)$. From the monotonicity of the sequences we can define the pointwise limits $\left(u_{\mathrm{s}}^{M}, v_{\mathrm{s}}^{M}\right)=\lim _{i \rightarrow \infty}\left(\bar{u}_{\mathrm{s}_{i}}, \bar{v}_{\mathrm{s}_{i}}\right)$ and $\left(u_{\mathrm{s}}^{m}, v_{\mathrm{s}}^{m}\right)=\lim _{i \rightarrow \infty}\left(\underline{u}_{\mathrm{S}_{i}}, \underline{v}_{\mathrm{s}_{i}}\right)$. From the continuity of $F$ and $G$, we then infer the pointwise convergence of $F_{i}=F\left(\bar{u}_{\mathrm{s}_{i}}, \bar{v}_{\mathrm{s}_{i}}\right)$ and $G_{i}=G\left(\bar{u}_{\mathrm{s}_{i}}, \bar{v}_{\mathrm{s}_{i}}\right)$ to $F\left(u_{\mathrm{s}}^{M}, v_{\mathrm{s}}^{M}\right)$ and $G\left(u_{\mathrm{s}}^{M}, v_{\mathrm{s}}^{M}\right)$ in the limit $i \rightarrow \infty$. Similarly we obtain the limits $F\left(u_{\mathrm{s}}^{m}, v_{\mathrm{s}}^{m}\right)=\lim _{i \rightarrow \infty} F\left(\underline{u}_{\mathrm{s}_{i}}, \underline{v}_{\mathrm{s}_{i}}\right)$ and $G\left(u_{\mathrm{s}}^{m}, v_{\mathrm{s}}^{m}\right)=\lim _{i \rightarrow \infty} G\left(\underline{u}_{\mathrm{s}_{i}}, \underline{v}_{\mathrm{s}_{i}}\right)$. This implies 
that $F_{i}$ and $G_{i}$ are uniformly bounded in $L^{p}(\Omega) \times L^{p}(\Omega)$ for any $p \geqslant 1$. From the Agmon-Douglis-Nirenberg estimates [1]:

$$
\left\|\vec{u}_{\mathrm{S}}\right\|_{\mathbf{W}_{0}^{2, p}(\Omega)} \leqslant C\|(F(x), G(x))\|_{\mathbf{L}^{p}(\Omega)} \leqslant C_{*},
$$

where $C_{*}$ is independent of $\left(\widetilde{u}_{\mathrm{s}}, \widetilde{v}_{\mathrm{s}}\right) \in\left\langle\underline{\vec{u}_{\mathrm{s}}}, \overrightarrow{\overrightarrow{u_{\mathrm{s}}}}\right\rangle$ and $\sigma$, and in which $\|(\cdot, \cdot)\|_{\mathbf{L}^{p}(\Omega)}$ and $\|(\cdot, \cdot)\|_{\mathbf{W}_{0}^{2, p}(\Omega)}$ are defined similarly to $|(\cdot, \cdot)|_{\mathbf{C}^{m+\alpha}(\bar{\Omega})}$. This implies that $\bar{u}_{\mathrm{S}_{i}}$ and $\bar{v}_{\mathrm{S}_{i}}$ are uniformly bounded in $\mathbf{W}_{0}^{2, p}(\Omega)$. By embedding, they are then uniformly bounded in $\mathbf{C}^{1+\alpha}(\bar{\Omega})$, which in turn implies that $F_{i}$ and $G_{i}$ are uniformly bounded in $\mathbf{C}^{\alpha}(\bar{\Omega})$ (from the Lipschitz properties of $f$ and $g$ ). From the Schauder estimates, (A.10), and the Arzela-Ascoli Theorem there exists a subsequence $\left(\bar{u}_{\mathrm{s}_{i_{k}}}, \bar{v}_{\mathrm{s}_{i_{k}}}\right)$ converging in $\mathbf{C}^{2}(\bar{\Omega})$ to $\left(u_{\mathrm{s}}^{\mathrm{M}}, v_{\mathrm{s}}^{\mathrm{M}}\right) \in \mathbf{C}^{2+\alpha}(\bar{\Omega})$ in the limit $k \rightarrow \infty$. But since $\left(u_{\mathrm{s}}^{M}, v_{\mathrm{s}}^{M}\right)=\lim _{i \rightarrow \infty}\left(\bar{u}_{\mathrm{s}_{i}}, \bar{v}_{\mathrm{s}_{i}}\right)$ pointwise, $\left(u_{\mathrm{s}}^{\mathrm{M}}, v_{\mathrm{s}}^{\mathrm{M}}\right)=\left(u_{\mathrm{s}}^{M}, v_{\mathrm{s}}^{M}\right) \in \mathbf{C}^{2+\alpha}(\bar{\Omega})$ and the limit of (A.9) is (6.1). By a similar argument we find that $\left(u_{\mathrm{s}}^{m}, v_{\mathrm{s}}^{m}\right)$ is also a classical solution. Moreover, if $\left(u_{\mathrm{s}}^{*}, v_{\mathrm{s}}^{*}\right)$ is any other solution in $\left\langle\underline{\vec{u}_{\mathrm{s}}}, \overline{\vec{u}_{\mathrm{s}}}\right\rangle$, then $\left(u_{\mathrm{s}}^{m}, v_{\mathrm{s}}^{m}\right) \preccurlyeq\left(u_{\mathrm{s}}^{*}, v_{\mathrm{s}}^{*}\right) \preccurlyeq\left(u_{\mathrm{s}}^{M}, v_{\mathrm{s}}^{M}\right)$ (see the method of the proof in [27, Theorem 8.4.1]).

\section{References}

[1] S. Agmon, A. Douglis, L. Nirenberg, Estimates near the boundary for solutions of elliptic partial differential equations satisfying general boundary conditions, Commun. Pure Appl. Math. 12 (1959) 623-727.

[2] R. Aris, The Mathematical Theory of Diffusion and Reaction in Permeable Catalysts, vol. II, Oxford University Press, London, 1975.

[3] E. Balakrishnan, A. Swift, G.C. Wake, Critical values for some non-class A geometries in thermal ignition theory, Math. Comput. Modelling 24 (1996) 1-10.

[4] J. Bebernes, D. Eberly, Mathematical problems from combustion theory, Applied Mathematical Science, vol. 83, Springer, New York, 1989.

[5] J. Bebernes, D. Kassoy, A mathematical analysis of blow-up for thermal runaway, SIAM J. Appl. Math. 40 (1981) $476-484$.

[6] H. Bellout, A criterion for blow-up of solutions to semilinear heat equations, SIAM J. Math. Anal. 18 (1987) 722-727.

[7] T. Boddington, P. Gray, G. Wake, Criteria for thermal explosions with and without reactant consumption, Proc. R. Soc. London A 357 (1977) 403-422.

[8] J. Brindley, J.F. Griffiths, A.C. McIntosh, J. Zhang, Effects of vaporization, diffusion and condensation on the combustion of reactive fluids in hot porous media, Proc. R. Soc. London A 456 (2000) 997-1017.

[9] J.G. Burnell, A.A. Lacey, G.C. Wake, Steady states of the reaction-diffusion equations. Parts I, II and III, J. Aust. Math. Soc. Ser. B 24 (1983) 374-391 24 (1983) 392-416; 27 (1985) 88-110.

[10] J. Carr, Applications of centre manifold theory, Applied Mathematical Sciences, vol. 35, Springer, New York, 1981.

[11] A. Constantin, J. Escher, Global solutions for quasilinear parabolic problems, J. Evol. Equations 2 (2002) 97-111.

[12] D.A. Frank-Kemenetskii, Diffusion and Heat Transfer in Chemical Kinetics, Princeton University Press, Princeton, NJ, 1955.

[13] A. Friedman, B. Mcleod, Blowup of positive solutions of semilinear heat equations, Indiana Univ. Math. J. 34 (1985) $425-477$.

[14] H. Fujita, On the nonlinear equations $\Delta u+e^{u}=0$ and $\partial v / \partial t=\Delta v+e^{v}$, Bull. Amer. Math. Soc. 75 (1969) 132-135.

[15] D. Gilbarg, N.S. Trudinger, Elliptic partial differential equations of second order, revised third ed., Classics in Mathematics, Springer, New York, 2001.

[16] B.F. Gray, G.C. Wake, Initial conditions for thermal ignition, Math. Comput. Modelling 18 (1993) 65-75.

[17] A.K. Kapila, Evolution of a deflagration in a cold combustible subjected to a uniform energy flux, Int. J. Eng. Sci. 19m (1981) 495-509.

[18] S. Kaplan, On the growth of solutions of quasilinear parabolic equations, Commun. Pure Appl. Math. 16 (1963) $327-330$.

[19] H.B. Keller, D.S. Cohen, Some positone problems suggested by nonlinear heat generation, J. Math. Mech. 16 (1967) $1361-1376$.

[20] A.A. Lacey, Mathematical analysis of thermal runaway for spatially inhomogeneous reactions, SIAM J. Appl. Math. 43 (1983) $1350-1366$.

[21] A.A. Lacey, D.E. Tzanetis, Global, unbounded solutions to a parabolic equation, J. Differential Equations 101 (1993) 80-102.

[22] O.A. Ladyzhenskaja, N.N. Ural'cera, Linear and Quasi-linear Elliptic Equations, Academic Press, New York, 1968.

[23] A. Liñan, F.A. Williams, Theory of ignition of a reactive solid by constant energy flux, Combust. Sci. Technol. 3 (1971) 91-98.

[24] S.B. Margolis, A.M. Telengator, F.A. Williams, Influence of subsurface gaseous combustion on the burning of confined porous energetic materials, Combust. Sci. Technol. 175 (2003) 421-452.

[25] G.N. Mercer, R.O. Weber, B.F. Gray, Combustion pseudo-waves, Math. Comput. Modelling 24 (1996) 29-38.

[26] G.N. Mercer, R.O. Weber, H.S. Sidhu, An oscillatory route to extinction for solid fuel combustion waves due to heat losses, Proc. R. Soc. London A 454 (1999) 2015-2022.

[27] C.V. Pao, Nonlinear Parabolic and Elliptic Equations, Plenum Press, New York, 1992.

[28] J. Petersson, On global existence for semilinear parabolic systems, Nonlinear Anal. 60 (2005) 337-347.

[29] M.H. Protter, H.F. Weinberger, Maximum Principles in Differential Equations, second ed., Springer, New York, 1984.

[30] D.H. Sattinger, Monotone methods in nonlinear parabolic and elliptic boundary value problems, Indiana Univ. Math. J. 21 (1972) 979-1000.

[31] D.H. Sattinger, Topics in Stability and Bifurcation Theory, Lecture Notes in Mathematics, vol. 309, Springer, New York, 1973.

[32] A. Shah, J. Brindley, J.F. Griffiths, A.C. McIntosh, The ignition of low-exothermicity solids by local heating, Trans. IChemE B 82 (B2) (2004) $1-15$.

[33] A. Shah, J. Brindley, A.C. McIntosh, J.F. Griffiths, The effects of gas production and movement on the ignition of porous solids, J. Eng. Math. (2007) to appear, 〈http://dx.doi.org/10.1007/s10665-006-9053-2〉. 
[34] A. Shah, G.C. Wake, The existence of steady states to a combustion model with internal heating, Nonlinear Anal. Real World Appl. 5 (3) (2004) 421-439.

[35] A.M. Telengator, S.B. Margolis, F.A. Williams, Ignition analysis of a porous energetic material: II. Ignition at a closed heated end, Combust. Theory Modelling 3 (3) (1999) 433-445.

[36] A.M. Telengator, S.B. Margolis, F.A. Williams, Analysis of ignition of a porous energetic material, Combust. Theory Modelling 3 (1) (1999) 33-49.

[37] F. Verhulst, Nonlinear Differential Equations and Dynamical Systems, Springer, Berlin, 1990.

[38] C.W. Wahle, B.J. Matkowsky, A.P. Aldushin, Effects of gas-solid nonequilibrium in filtration combustion, Combust. Sci. Technol. 175 (2003) $1389-1499$.

[39] R.O. Weber, G.N. Mercer, H.S. Sidhu, B.F. Gray, Combustion waves for gases $(L e=1)$ and solids $(L e \rightarrow \infty)$, Proc. R. Soc. London A 453 (1997) 1105-1118 